TITLE:

\title{
Singularity of the Velocity Distribution Function in Molecular Velocity Space
}

\section{$\operatorname{AUTHOR}(\mathrm{S})$ :}

Chen, I. Kun; Funagane, Hitoshi; Liu, Tai Ping;

Takata, Shigeru

\section{CITATION:}

Chen, I. Kun ... [et al]. Singularity of the Velocity Distribution Function in Molecular Velocity Space. Communications in Mathematical Physics 2016, 341: 105-134

\section{ISSUE DATE:}

2016-01

URL:

http://hdl.handle.net/2433/261180

\section{RIGHT:}

This is a post-peer-review, pre-copyedit version of an article published in Communications in Mathematical Physics. The final authenticated version is available online at: http://dx.doi.org/10.1007/s00220-015-2476-8;; この論文は出版社 版でありません。引用の際には出版社版をご確認じ利用ください。; This is not the published version. Please cite only the published version. 


\title{
Singularity of the velocity distribution function in
}

\section{molecular velocity space}

\author{
I-Kun Chen ${ }^{1}$, Hitoshi Funagane ${ }^{2}$, Tai-Ping Liu ${ }^{3}$, Shigeru Takata ${ }^{4}$ \\ 1 Department of Applied Analysis, Graduate School of Informatics, Kyoto University, Kyoto \\ 606-8501, Japan \\ E-mail: ikun.chen@acs.i.kyoto-u.ac.jp \\ 2 Department of Mechanical Engineering and Science, Kyoto University, Kyoto 606-8501, \\ Japan \\ E-mail: hitoshi.funagane@gmail.com \\ 3 Institute of Mathematics, Academia Sinica, Taipei, ROC and Department of Mathematics, \\ Stanford University, USA \\ E-mail: liu@math.stanford.edu \\ 4 Department of Mechanical Engineering and Science and Advanced Research Institute of \\ Fluid Science and Engineering, Kyoto University, Kyoto 606-8501, Japan \\ E-mail: takata.shigeru.4a@kyoto-u.ac.jp
}

Received: date / Accepted: date

\begin{abstract}
We study the boundary singularity of the solutions to the Boltzmann equation in the kinetic theory. The solution has a jump discontinuity in the microscopic velocity $\zeta$ on the boundary and a secondary singularity of logarithmic type around the velocity tangential to the boundary, $\zeta_{n} \sim 0_{-}$, where $\zeta_{n}$ is the component of molecular velocity normal to the boundary, pointing to the gas. We demonstrate this secondary singularity by obtaining an asymptotic formula for the derivative of the solution on the boundary with respect to $\zeta_{n}$ that diverges logarithmically when $\zeta_{n} \sim 0_{-}$. Our study is for the thermal tran-
\end{abstract}


spiration problem between two plates for the hard sphere gases with sufficiently large Knudsen number and with the diffuse reflection boundary condition. The solution is constructed and its singularity is studied by an iteration procedure.

\section{Introduction}

The kinetic theory allows for rich boundary phenomena. In the present paper, we are interested in the singularity of the velocity distribution function on the boundary, which is an important element of the boundary phenomena. We consider the problem for thermal transpiration between two plates with diffuse reflection boundary condition. It is known in [5] that the velocity distribution function on the boundary has a jump discontinuity at $\zeta_{n}=0$, where $\zeta_{n}$ is the normal component of the molecular velocity at the boundary, pointing to the gas. A more subtle secondary singularity is in the form of $\zeta_{n} \ln \left|\zeta_{n}\right|$ for $\zeta_{n} \sim 0_{-}$, [7]. In the present paper, for highly rarefied gases, we confirm the secondary singularity by obtaining an asymptotic formula for the derivative of the solution on the boundary with respect to $\zeta_{n}$ that diverges logarithmically when $\zeta_{n} \sim 0_{-}$.

Consider stationary linearized Boltzmann equation:

$$
\sum_{i=1}^{3} \zeta_{i} \partial_{x_{i}} f(x, \zeta)=\frac{1}{\kappa} L(f)(x, \zeta),
$$

in a region between two parallel infinite plates

$$
\Omega=\left\{x \in \mathbb{R}^{3}:-\frac{1}{2}<x_{1}<\frac{1}{2}\right\} .
$$

The linearization is respect to the normalized Maxwellian

$$
E(\zeta)=\pi^{-\frac{3}{2}} e^{-|\zeta|^{2}} .
$$


The diffuse reflection boundary condition prescribes that the distribution leaving the boundary is in thermal equilibrium with the boundary temperature and that there is no mass flux across the boundary; for the domain we consider,

$$
\begin{aligned}
& f\left( \pm \frac{1}{2}, x_{2}, x_{3}, \zeta\right)=\sigma_{ \pm} E^{\frac{1}{2}}+\tau_{w}\left(|\zeta|^{2}-\frac{3}{2}\right) E^{\frac{1}{2}}, \quad \text { for } \zeta_{1} \lessgtr 0 \\
& \sigma_{ \pm}=-\frac{1}{2} \tau_{w}+2 \sqrt{\pi} \int_{\zeta_{1} \gtrless 0}\left|\zeta_{1}\right| E^{\frac{1}{2}} f\left( \pm \frac{1}{2}, x_{2}, x_{3}, \zeta\right) d \zeta
\end{aligned}
$$

where $\tau_{w}$ is the perturbation of the boundary temperature around the reference normalized temperature. The thermal transpiration phenomenon is that temperature gradient of the boundary, without pressure gradient, can induce a gas flow. The problem is considered here with linear boundary temperature, $\tau_{w}=c x_{2}$, so that the solution can take a special form:

$$
f(x, \zeta)=c\left(\tilde{\phi}\left(x_{1}, \zeta\right)+x_{2}\left(|\zeta|^{2}-\frac{5}{2}\right) E^{\frac{1}{2}}\right)
$$

and the symmetric part of $\tilde{\phi}$ defined by

$$
\phi\left(x_{1}, \zeta_{1}, \zeta_{2}, \zeta_{3}\right):=\frac{1}{2}\left(\tilde{\phi}\left(x_{1}, \zeta_{1}, \zeta_{2}, \zeta_{3}\right)+\tilde{\phi}\left(-x_{1},-\zeta_{1}, \zeta_{2}, \zeta_{3}\right)-\left(\sigma_{+}+\sigma_{-}\right) E^{\frac{1}{2}}\right),
$$

vanishes on the boundary for molecular velocity toward the gas region:

$$
\begin{cases}\zeta_{1} \partial_{x_{1}} \phi\left(x_{1}, \zeta\right)=\frac{1}{\kappa} L(\phi)-\zeta_{2}\left(|\zeta|^{2}-\frac{5}{2}\right) E^{\frac{1}{2}}(\zeta), & \text { for }-\frac{1}{2}<x_{1}<\frac{1}{2} \\ \phi\left( \pm \frac{1}{2}, \zeta\right)=0, & \text { for } \zeta_{1} \lessgtr 0 .\end{cases}
$$

Here, we can observe that $\phi$ on the boundary has a jump at $\zeta_{1}=0$ whenever it is not zero for the microscopic velocity toward the boundary and close to tangential to the boundary. The linear collision operator can be decomposed into a damping multiplicative operator $-\nu$ and a smoothing integral operator $K$ :

$$
L(\phi)(\zeta)=-\nu(|\zeta|) \phi(\zeta)+K(\phi)(\zeta)
$$


For the hard sphere case considered here,

$$
\begin{aligned}
\nu(\eta)= & 2^{-3 / 2}\left(e^{-\eta^{2}}+\left(2 \eta+\frac{1}{\eta}\right) \int_{0}^{\eta} e^{-s^{2}} d s\right), \\
K(\phi)(\zeta)= & \int_{\mathbb{R}^{3}} k\left(\zeta, \zeta_{*}\right) \phi\left(\zeta_{*}\right) d \zeta_{*}, \\
k\left(\zeta, \zeta_{*}\right)= & 2^{-\frac{1}{2}} \pi^{-1}\left|\zeta-\zeta_{*}\right|^{-1} e^{-\frac{1}{4} \frac{\left(|\zeta|^{2}-\left|\zeta_{*}\right|^{2}\right)^{2}}{\left|\zeta-\zeta_{*}\right|^{2}}-\frac{1}{4}\left|\zeta-\zeta_{*}\right|^{2}} \\
& -2^{-\frac{3}{2}} \pi^{-1}\left|\zeta-\zeta_{*}\right| e^{-\frac{|\zeta|^{2}}{2}-\frac{\left|\zeta_{*}\right|^{2}}{2}} \\
= & k_{1}\left(\zeta, \zeta_{*}\right)-k_{2}\left(\zeta, \zeta_{*}\right),
\end{aligned}
$$

and the following bound for $\nu$ is well known: there exist positive constants $\nu_{0}$ and $\nu_{1}$ such that

$$
\nu_{0}(1+|\zeta|)<\nu(|\zeta|)<\nu_{1}(1+|\zeta|) .
$$

We will view the smoothing term $K(\phi)$ as the source in (1.5) and integrate (1.5) to obtain an integral equation for $\phi$ :

$$
\begin{aligned}
& \phi\left(x_{1}, \zeta\right)= \\
& \left\{\begin{array}{l}
\int_{-\frac{1}{2}}^{x_{1}} \frac{1}{\left|\zeta_{1}\right|} e^{-\frac{\nu(|\zeta|) \mid}{\kappa\left|\zeta_{1}\right|}\left|x_{1}-s\right|}\left(\frac{1}{\kappa} K(\phi)(s, \zeta)-\zeta_{2}\left(|\zeta|^{2}-\frac{5}{2}\right) E^{\frac{1}{2}}(\zeta)\right) d s, \text { for } \zeta_{1}>0, \\
\int_{x_{1}}^{\frac{1}{2}} \frac{1}{\left|\zeta_{1}\right|} e^{-\frac{\nu(|\zeta|)}{\kappa\left|\zeta_{1}\right|}\left|x_{1}-s\right|}\left(\frac{1}{\kappa} K(\phi)(s, \zeta)-\zeta_{2}\left(|\zeta|^{2}-\frac{5}{2}\right) E^{\frac{1}{2}}(\zeta)\right) d s, \text { for } \zeta_{1}<0 .
\end{array}\right.
\end{aligned}
$$

To solve (1.11), we consider an iteration scheme:

$$
\begin{aligned}
& \phi_{0}\left(x_{1}, \zeta\right)=\left\{\begin{array}{l}
\frac{\kappa}{\nu}\left(1-e^{-\frac{\nu}{\kappa\left|\zeta_{1}\right|}\left(\frac{1}{2}+x_{1}\right)}\right) \zeta_{2}\left(\frac{5}{2}-|\zeta|^{2}\right) E^{\frac{1}{2}}, \text { for } \zeta_{1}>0, \\
\frac{\kappa}{\nu}\left(1-e^{-\frac{\nu}{\kappa\left|\zeta_{1}\right|}\left(\frac{1}{2}-x_{1}\right)}\right) \zeta_{2}\left(\frac{5}{2}-|\zeta|^{2}\right) E^{\frac{1}{2}}, \text { for } \zeta_{1}<0,
\end{array}\right. \\
& \phi_{n}\left(x_{1}, \zeta\right)=\left\{\begin{array}{l}
\int_{-\frac{1}{2}}^{x_{1}} \frac{1}{\kappa\left|\zeta_{1}\right|} e^{-\frac{\nu}{\kappa\left|\zeta_{1}\right|}\left|x_{1}-s\right|} K\left(\phi_{n-1}\right) d s, \text { for } \zeta_{1}>0, \\
\int_{x_{1}}^{\frac{1}{2}} \frac{1}{\kappa\left|\zeta_{1}\right|} e^{-\frac{\nu}{\kappa\left|\zeta_{1}\right|}\left|x_{1}-s\right|} K\left(\phi_{n-1}\right) d s, \text { for } \zeta_{1}<0 .
\end{array}\right.
\end{aligned}
$$

In the above, $\nu=\nu(|\zeta|)$ and $K(\phi)=K(\phi)(s, \zeta)$; such abbreviations will be used in what follows. For a highly rarefied gas, $\kappa$ large enough,

$$
\phi=\sum_{n=0}^{\infty} \phi_{n}
$$


converges and solves (1.11), [2]. A numerical method is developed in [6] based on this iteration. In [3], it is used to prove the logarithmic singularity of flow velocity near the boundary. It is indicated in [7] that the singularity in $x_{1}$ of logarithmic type of $K(\phi)$ induces the same type of singularity in $\zeta_{1}$ on the boundary. This observation is a key element in the present study.

In the present paper, we prove that the divergence of $\partial_{x_{1}} K(\phi)\left(x_{1}, \zeta\right)$ near upper (lower) boundary as a logarithmic function induces the same type of divergence of $\partial_{\zeta_{1}} \phi$ on the upper (lower) boundary for $\zeta_{1} \sim 0_{+}\left(0_{-}\right)$. The main step is to prove that $\partial_{x_{1}} K\left(\phi_{0}\right)$ has the singularity, and this task is reduced to analyzing an integral $\bar{F}$, a function of $\zeta_{1}$ and $\zeta_{r}=\left(\zeta_{2}^{2}+\zeta_{3}^{2}\right)^{\frac{1}{2}}$ defined in Definition 1. It is known from [3] that the higher order terms $\partial_{x_{1}} K\left(\phi_{n}\right), n \geq 1$ can be controlled when $\kappa$ is sufficiently large.

In our theorem, we characterize the secondary singularity by the following asymptotic formula for $0<\zeta_{1}<\frac{1}{\kappa^{2}}<\frac{1}{100}$.

Theorem 1.1. If $\kappa$ large enough and $0<\zeta_{1}<\frac{1}{\kappa^{2}}<\frac{1}{100}$, then

$$
\partial_{\zeta_{1}} \phi\left(\frac{1}{2}, \zeta\right)=\frac{\kappa}{\nu^{2}}\left\{-\ln \left(\frac{\kappa\left|\zeta_{1}\right|}{\nu}\right)\left[\zeta_{2} U\left(\zeta_{r}\right)+O\left(\frac{\ln \kappa}{\kappa}\right)\right]+O(1)\right\}
$$

where $\zeta_{r}=\sqrt{\zeta_{2}^{2}+\zeta_{3}^{2}}$ and

$$
U\left(\zeta_{r}\right):=-2^{-\frac{1}{2}} \pi^{-\frac{1}{4}}\left(1-\frac{3}{8} \zeta_{r}^{2}\right) e^{-\frac{1}{2} \zeta_{r}^{2}}-2^{-\frac{7}{4}} \pi^{-\frac{1}{4}} I_{0}\left(\frac{1}{2} \zeta_{r}^{2}\right) e^{-\zeta_{r}^{2}}
$$

Here, $I_{0}$ is the zeroth order modified Bessel function of the the first kind.

Remark 1.1. In [7], from the symmetry of the equation, the authors observe that $\phi E^{\frac{1}{2}} / \zeta_{2}=\bar{\phi}\left(s, \zeta_{1},|\zeta|\right)$. They confirmed numerically

$$
\bar{\phi}\left(\frac{1}{2}, \zeta_{1},|\zeta|\right) \approx a_{1}+a_{2} \zeta_{1} \ln \zeta_{1}, \quad \text { for } \zeta_{1} \sim 0_{+},
$$


where $a_{1}$ and $a_{2}$ are independent of $\zeta_{1}$. To see the correspondence between Theorem 1.1 and (1.17), we introduce new variables $\mu, \rho, \theta$ that represent $\zeta$ by

$$
\zeta=\left(\mu, \sqrt{\rho^{2}-\mu^{2}} \cos \theta, \sqrt{\rho^{2}-\mu^{2}} \sin \theta\right),
$$

to have

$$
\begin{aligned}
\zeta_{2} \frac{\partial}{\partial \mu} \bar{\phi}\left(\frac{1}{2}, \mu, \rho\right)= & E^{\frac{1}{2}} \frac{\partial}{\partial \zeta_{1}} \phi\left(\frac{1}{2}, \zeta\right)+\frac{\zeta_{1} \cos \theta}{\sqrt{\zeta_{2}^{2}+\zeta_{3}^{2}}} \bar{\phi}\left(\frac{1}{2}, \mu, \rho\right) \\
& -E^{\frac{1}{2}}\left(\frac{\zeta_{1} \cos \theta}{\sqrt{\zeta_{2}^{2}+\zeta_{3}^{2}}} \frac{\partial}{\partial \zeta_{2}} \phi\left(\frac{1}{2}, \zeta\right)+\frac{\zeta_{1} \sin \theta}{\sqrt{\zeta_{2}^{2}+\zeta_{3}^{2}}} \frac{\partial}{\partial \zeta_{3}} \phi\left(\frac{1}{2}, \zeta\right)\right) .
\end{aligned}
$$

For fixed $\rho>0$, say $\rho=0.5$, and $0<\zeta_{1}<\frac{1}{\kappa^{2}}<\frac{1}{100}$, the second term on the right hand side of the equation above is bounded from the numerical computation and the last term is bounded by $C \frac{\kappa}{\nu^{2}} E^{\frac{1}{2}}$ to be studied in Section 5 . Therefore, we conclude that Theorem 1.1 and (1.17) are consistent. Numerical demonstration of (1.17) for $\rho=0.5$ can be found in Figure 5 of [7].

In the next section, we lay out an overall plan for the proof of Theorem 1.1. In Section 3 , an asymptotic formula with spatial singularity of $\partial_{x_{1}} K\left(\phi_{0}\right)$ is studied. The exact expression of the function $\bar{F}\left(0, \zeta_{r}\right)$ and the continuity of $\bar{F}$ for $\zeta_{1} \sim 0$ is studied in Section 4. The contribution of the higher order terms $\partial_{x_{1}} K\left(\phi_{n}\right), n \geq 1$ is estimated in Section 5.

Our analysis of the boundary singularity for the Boltzmann equation consists of exact calculations of the solution for the particular physical situation of thermal transpiration in the parallel plates. We anticipate that this type of singularity demonstrated here would happen for more general situations.

\section{Plan of proof}

We will lay out the plan of proof of Theorem 1.1 in this section. 
We formally differentiate $\phi_{n}, n \geq 1$, in (1.13) with respect to $\zeta_{1}$ on the boundary, say, at the upper boundary, $x_{1}=\frac{1}{2}$ :

$$
\partial_{\zeta_{1}} \phi_{n}\left(\frac{1}{2}, \zeta\right)= \begin{cases}-\int_{-\frac{1}{2}}^{\frac{1}{2}} \frac{1}{\kappa\left|\zeta_{1}\right|^{2}}\left(1-\frac{\nu}{\kappa\left|\zeta_{1}\right|}\left|\frac{1}{2}-s\right|\right) e^{-\frac{\nu}{\kappa\left|\zeta_{1}\right| \frac{1}{2}-s \mid} K\left(\phi_{n-1}\right) d s} \\ +\int_{-\frac{1}{2}}^{\frac{1}{2}} \frac{1}{\kappa\left|\zeta_{1}\right|} e^{-\frac{\nu}{\kappa\left|\zeta_{1}\right|}\left|\frac{1}{2}-s\right|} \partial_{\zeta_{1}} K\left(\phi_{n-1}\right) d s & \\ -\int_{-\frac{1}{2}}^{\frac{1}{2}} \frac{1}{\kappa\left|\zeta_{1}\right|} e^{-\frac{\nu}{\kappa\left|\zeta_{1}\right|}\left|\frac{1}{2}-s\right|} \frac{\nu^{\prime}\left|\frac{1}{2}-s\right|}{\kappa|\zeta|} K\left(\phi_{n-1}\right) d s, & \zeta_{1}>0, \\ 0, & \zeta_{1}<0,\end{cases}
$$

where $\nu^{\prime}=\left.\frac{d \nu(\rho)}{d \rho}\right|_{\rho=|\zeta|}$. The integration by parts of the first term simplifies the expression for $\zeta_{1}>0$ as

$$
\begin{aligned}
\partial_{\zeta_{1}} \phi_{n}\left(\frac{1}{2}, \zeta\right)= & -\frac{1}{\kappa\left|\zeta_{1}\right|^{2}} \int_{-\frac{1}{2}}^{\frac{1}{2}} e^{-\frac{\nu}{\kappa\left|\zeta_{1}\right|}\left(\frac{1}{2}-s\right)}\left(\frac{1}{2}-s\right) \partial_{s} K\left(\phi_{n-1}\right) d s \\
& -\frac{1}{\kappa\left|\zeta_{1}\right|^{2}} e^{-\frac{\nu}{\kappa\left|\zeta_{1}\right|} K\left(\phi_{n-1}\right)\left(-\frac{1}{2}, \zeta\right)} \\
& -\int_{-\frac{1}{2}}^{\frac{1}{2}} \frac{1}{\kappa\left|\zeta_{1}\right|} e^{-\frac{\nu}{\kappa \mid \zeta_{1}}\left|\frac{1}{2}-s\right|} \frac{\nu^{\prime}\left|\frac{1}{2}-s\right|}{\kappa|\zeta|} K\left(\phi_{n-1}\right) d s \\
& +\int_{-\frac{1}{2}}^{\frac{1}{2}} \frac{1}{\kappa\left|\zeta_{1}\right|} e^{-\frac{\nu}{\kappa \mid \zeta_{1}}\left|\frac{1}{2}-s\right|} \partial_{\zeta_{1}} K\left(\phi_{n-1}\right) d s .
\end{aligned}
$$

Our goal is to show that the logarithmic singularity of $\partial_{\zeta_{1}} \phi$ is dominated by the first term on the right hand side of (2.1) for $n=1$ :

$$
W:=\frac{1}{\kappa\left|\zeta_{1}\right|^{2}} \int_{-\frac{1}{2}}^{\frac{1}{2}} e^{-\frac{\nu}{\kappa\left|\zeta_{1}\right|}\left(\frac{1}{2}-s\right)}\left(\frac{1}{2}-s\right) \partial_{s} K\left(\phi_{0}\right) d s .
$$

The main source of singularity of $W$ is the spatial singularity of $\partial_{s} K\left(\phi_{0}\right) \sim$ $-\ln \left(\frac{1}{2}-s\right)$. The following lemma shows that the spatial singularity of $\ln \left(\frac{1}{2}-s\right)$ type induces a singularity in $\zeta_{1}$ for $W,(2.2)$.

\section{Lemma 2.1 .}

$\int_{-\frac{1}{2}}^{\frac{1}{2}} \frac{1}{\kappa\left|\zeta_{1}\right|^{2}} e^{-\frac{\nu}{\kappa\left|\zeta_{1}\right|}\left(\frac{1}{2}-s\right)}\left(\frac{1}{2}-s\right) \ln \left(\frac{1}{2}-s\right) d s=\frac{\kappa}{\nu^{2}}\left(\ln \frac{\kappa\left|\zeta_{1}\right|}{\nu}+1-\gamma+O\left(e^{-\frac{\nu}{2 \kappa\left|\zeta_{1}\right|}}\right)\right)$, where $\gamma$ is the Euler constant. 
Proof. Letting $z=\frac{\nu(|\zeta|)}{\kappa\left|\zeta_{1}\right|}\left(\frac{1}{2}-s\right)$, we have

$$
\begin{aligned}
& \int_{-\frac{1}{2}}^{\frac{1}{2}} \frac{1}{\kappa\left|\zeta_{1}\right|^{2}} e^{-\frac{\nu}{\kappa\left|\zeta_{1}\right|}\left(\frac{1}{2}-s\right)}\left(\frac{1}{2}-s\right) \ln \left(\frac{1}{2}-s\right) d s \\
& =\frac{\kappa}{\nu^{2}}\left(\int_{0}^{\frac{\nu}{\kappa\left|\zeta_{1}\right|}} e^{-z} z \ln z d z+\ln \frac{\kappa\left|\zeta_{1}\right|}{\nu} \int_{0}^{\frac{\nu}{\kappa\left|\zeta_{1}\right|}} e^{-z} z d z\right) \\
& =\frac{\kappa}{\nu^{2}}\left(1-\gamma-\int_{\frac{\nu}{\kappa\left|\zeta_{1}\right|}}^{\infty} e^{-z} z \ln z d z+\ln \frac{\kappa\left|\zeta_{1}\right|}{\nu}\left(1-\frac{\nu}{\kappa\left|\zeta_{1}\right|} e^{-\frac{\nu}{\kappa\left|\zeta_{1}\right|}}-e^{-\frac{\nu}{\kappa \mid \zeta_{1}}}\right)\right) \\
& =\frac{\kappa}{\nu^{2}}\left(\ln \frac{\kappa\left|\zeta_{1}\right|}{\nu}+1-\gamma+O\left(e^{-\frac{\nu}{2 \kappa \mid \zeta_{1}}}\right)\right) .
\end{aligned}
$$

The first step in studying the spatial singularity of $\partial_{s} K\left(\phi_{0}\right)$ is to derive the following asymptotic formula.

\section{Lemma 2.2.}

$$
\partial_{s} K\left(\phi_{0}\right)(s, \zeta)=\left(\ln \left(\frac{1}{2}+s\right)-\ln \left(\frac{1}{2}-s\right)\right)\left(\zeta_{2} \bar{F}\left(\zeta_{1}, \zeta_{r}\right)+O\left(\frac{1}{\kappa^{2}}\right)\right)+O(1)
$$

Here, $\zeta_{r}=\left(\zeta_{2}^{2}+\zeta_{3}^{2}\right)^{\frac{1}{2}}$ and $\bar{F}$ is a function defined later in Definition 1.

Lemma 2.2 will be proved in Section 3, Lemma 3.1 and Section 4, Lemma 4.1. It implies that $\partial_{s} K\left(\phi_{0}\right)$ has a logarithmic singularity in $s$ as long as $\zeta_{2} \bar{F}$ is bounded away from zero. In Section 4, the following closed form expression for $\bar{F}\left(0, \zeta_{r}\right)$ and the continuity of $\bar{F}$ when $\zeta_{1} \sim 0$ will be obtained.

\section{Lemma 2.3.}

$$
\bar{F}\left(0, \zeta_{r}\right)=-2^{-\frac{1}{2}} \pi^{-\frac{1}{4}}\left(1-\frac{3}{8} \zeta_{r}^{2}\right) e^{-\frac{\zeta_{r}^{2}}{2}}-2^{-\frac{7}{4}} \pi^{-\frac{1}{4}} I_{0}\left(\frac{1}{2} \zeta_{r}^{2}\right) e^{-\zeta_{r}^{2}},
$$

where $I_{0}(x)$ is the zeroth order modified Bessel function of the first kind.

Lemma 2.4. If $0 \leq \bar{\zeta}_{1} \leq \zeta_{1} \leq \frac{1}{100}$, then

$$
\left|\bar{F}\left(\zeta_{1}, \zeta_{r}\right)-\bar{F}\left(\bar{\zeta}_{1}, \zeta_{r}\right)\right| \leq C\left|\zeta_{1}-\bar{\zeta}_{1}\right|^{\frac{1}{2}}
$$


The remaining terms in (2.1) for $n=1$ are minor and studied in Section 5 . Also, the higher order terms, i.e., (2.1) for $n=2,3, \ldots$, are minor, which is also discussed in Section 5. Summarizing the contributions of these minor terms, we have

Lemma 2.5. For large enough $\kappa$ and $0<\zeta_{1}<\frac{1}{\kappa^{2}}$,

$$
\begin{aligned}
\left|\partial_{\zeta_{1}} \phi\left(\frac{1}{2}, \zeta\right)-\partial_{\zeta_{1}} \phi_{1}\left(\frac{1}{2}, \zeta\right)-\partial_{\zeta_{1}} \phi_{0}\left(\frac{1}{2}, \zeta\right)\right| & \leq C \frac{\kappa}{\nu^{2}}\left(\left|\ln \frac{\kappa \zeta_{1}}{\nu}\right|+\ln \kappa\right)\left(\frac{C \ln \kappa}{\kappa}\right), \\
\left|\partial_{\zeta_{1}} \phi_{1}\left(\frac{1}{2}, \zeta\right)+W\right| & <\frac{C \kappa}{\nu^{2}} \\
\left|\partial_{\zeta_{1}} \phi_{0}\left(\frac{1}{2}, \zeta\right)\right| & \leq \frac{C \kappa}{\nu^{2}}
\end{aligned}
$$

With the lemmas above as given, we can present the proof of Theorem 1.1.

Proof of Theorem 1.1. Let $U\left(\zeta_{r}\right)=\bar{F}\left(0, \zeta_{r}\right)$. Combining Lemmas 2.2, 2.3, and 2.4 , we obtain

$$
\partial_{s} K\left(\phi_{0}\right)(s, \zeta)=\left(\ln \left(\frac{1}{2}+s\right)-\ln \left(\frac{1}{2}-s\right)\right)\left(\zeta_{2} U\left(\zeta_{r}\right)+O\left(\frac{1}{\kappa}\right)\right)+O(1),
$$

for $0 \leq \zeta_{1} \leq \frac{1}{\kappa^{2}} \leq \frac{1}{100}$. Here we have used that $\zeta_{1}^{\frac{1}{2}} \leq \frac{1}{\kappa}$.

Similar to Lemma 2.1, we have

$$
\int_{0}^{\frac{1}{2}} \frac{1}{\kappa\left|\zeta_{1}\right|^{2}} e^{-\frac{\nu}{\kappa\left|\zeta_{1}\right|}\left(\frac{1}{2}-s\right)}\left(\frac{1}{2}-s\right) \ln \left(\frac{1}{2}-s\right) d s=\frac{\kappa}{\nu^{2}}\left(\ln \frac{\kappa \zeta_{1}}{\nu}+1-\gamma+O\left(e^{-\frac{\nu}{4 \kappa\left|\zeta_{1}\right|}}\right)\right) .
$$

Furthermore,

$$
\begin{aligned}
& \left|\int_{0}^{\frac{1}{2}} \frac{1}{\kappa\left|\zeta_{1}\right|^{2}} e^{-\frac{\nu}{\kappa\left|\zeta_{1}\right|}\left(\frac{1}{2}-s\right)}\left(\frac{1}{2}-s\right)\left(\left|\ln \left(\frac{1}{2}+s\right)\right|+C\right) d s\right| \\
& \leq \int_{0}^{\frac{1}{2}} \frac{1}{\kappa\left|\zeta_{1}\right|^{2}} e^{-\frac{\nu}{\kappa\left|\zeta_{1}\right|}\left(\frac{1}{2}-s\right)}\left(\frac{1}{2}-s\right)(\ln 2+C) d s \leq C \frac{\kappa}{\nu^{2}},
\end{aligned}
$$

and

$$
\begin{aligned}
& \int_{-\frac{1}{2}}^{0} \frac{1}{\kappa\left|\zeta_{1}\right|^{2}} e^{-\frac{\nu}{\kappa\left|\zeta_{1}\right|}\left(\frac{1}{2}-s\right)}\left(\frac{1}{2}-s\right)\left(\left|\ln \left(\frac{1}{2}+s\right)\right|+\left|\ln \left(\frac{1}{2}-s\right)\right|+C\right) d s \\
& \leq \int_{-\frac{1}{2}}^{0} \frac{1}{\kappa\left|\zeta_{1}\right|^{2}} e^{-\frac{\nu}{2 \kappa\left|\zeta_{1}\right|}}\left(\left|\ln \left(\frac{1}{2}+s\right)\right|+\ln 2+C\right) d s \leq C \frac{\kappa}{\nu^{2}} .
\end{aligned}
$$


Combining the inequalities above together, for small $\zeta_{1}>0$, we have an asymptotic formula for $W$ of $(2.2)$ :

$$
W=\frac{\kappa}{\nu^{2}}\left\{\ln \left(\frac{\kappa\left|\zeta_{1}\right|}{\nu}\right)\left[\zeta_{2} U\left(\zeta_{r}\right)+O\left(\frac{1}{\kappa}\right)\right]+O(1)\right\}
$$

Combining (2.10) with Lemma 2.5, for large enough $\kappa$, we obtain (1.15) and complete the proof of Theorem 1.1.

\section{Spatial singularity of $\partial_{s} K\left(\phi_{0}\right)(s, \zeta)$}

The goal of this section is to prove the following lemma:

Lemma 3.1. If $\kappa$ is large enough, then

$$
\partial_{s} K\left(\phi_{0}\right)(s, \zeta)=\left(\ln \left(\frac{1}{2}+s\right)-\ln \left(\frac{1}{2}-s\right)\right)\left(F(\zeta)+O\left(\frac{1}{\kappa^{2}}\right)\right)+O(1)
$$

where

$$
\begin{aligned}
F(\zeta) & :=-\int_{0}^{2 \pi} \int_{0}^{\infty} g_{\zeta}(\rho, 0, \varphi) d \rho d \varphi+e^{-\frac{|\zeta|^{2}}{2}} \int_{0}^{2 \pi} \int_{0}^{\infty} h_{\zeta}(\rho, 0, \varphi) d \rho d \varphi \\
g_{\zeta}(\rho, \alpha, \varphi) & :=c_{1}\left|\zeta-\zeta_{*}\right|^{-1} e^{-\frac{\left(|\zeta|^{2}-\rho^{2}\right)^{2}}{4\left|\zeta-\zeta_{*}\right|^{2}}-\frac{1}{4}\left|\zeta-\zeta_{*}\right|^{2}} \sqrt{1-\alpha^{2}} \cos \varphi\left(\frac{5}{2}-\rho^{2}\right) e^{-\frac{\rho^{2}}{2}} \rho^{2} \\
h_{\zeta}(\rho, \alpha, \varphi) & :=c_{2}\left|\zeta-\zeta_{*}\right| \sqrt{1-\alpha^{2}} \cos \varphi\left(\frac{5}{2}-\rho^{2}\right) e^{-\rho^{2}} \rho^{2}
\end{aligned}
$$

and

$$
\begin{gathered}
c_{1}=2^{-\frac{1}{2}} \pi^{-\frac{7}{4}}, \quad c_{2}=2^{-\frac{3}{2}} \pi^{-\frac{7}{4}} \\
\left|\zeta-\zeta_{*}\right|=\left(\left(\zeta_{1}-\rho \alpha\right)^{2}+\left(\zeta_{2}-\rho \sqrt{1-\alpha^{2}} \cos \varphi\right)^{2}+\left(\zeta_{3}-\rho \sqrt{1-\alpha^{2}} \sin \varphi\right)^{2}\right)^{\frac{1}{2}}
\end{gathered}
$$


Proof. Substitution of (1.12) into the definition of $K,(1.8)$ and (1.9), yields

$$
\begin{aligned}
\partial_{s} K\left(\phi_{0}\right)= & -\int_{\zeta_{* 1}<0} \pi^{-\frac{3}{4}} k_{1}\left(\zeta, \zeta_{*}\right) \frac{1}{\left|\zeta_{* 1}\right|} e^{-\frac{\nu_{*}}{\kappa \mid \zeta_{* 1}}\left(\frac{1}{2}-s\right)} \zeta_{* 2}\left(\frac{5}{2}-\left|\zeta_{*}\right|^{2}\right) e^{-\frac{1}{2}\left|\zeta_{*}\right|^{2}} d \zeta_{*} \\
& +\int_{\zeta_{* 1}<0} \pi^{-\frac{3}{4}} k_{2}\left(\zeta, \zeta_{*}\right) \frac{1}{\left|\zeta_{* 1}\right|} e^{-\frac{\nu_{*}}{\kappa \mid \zeta_{* 1}}\left(\frac{1}{2}-s\right)} \zeta_{* 2}\left(\frac{5}{2}-\left|\zeta_{*}\right|^{2}\right) e^{-\frac{1}{2}\left|\zeta_{*}\right|^{2}} d \zeta_{*} \\
& +\int_{\zeta_{* 1}>0} \pi^{-\frac{3}{4}} k_{1}\left(\zeta, \zeta_{*}\right) \frac{1}{\left|\zeta_{* 1}\right|} e^{-\frac{\nu_{*}}{\kappa \mid \zeta_{* 1}}\left(\frac{1}{2}+s\right)} \zeta_{* 2}\left(\frac{5}{2}-\left|\zeta_{*}\right|^{2}\right) e^{-\frac{1}{2}\left|\zeta_{*}\right|^{2}} d \zeta_{*} \\
& -\int_{\zeta_{* 1}>0} \pi^{-\frac{3}{4}} k_{2}\left(\zeta, \zeta_{*}\right) \frac{1}{\left|\zeta_{* 1}\right|} e^{-\frac{\nu_{*}}{\kappa \mid \zeta_{* 1}}\left(\frac{1}{2}+s\right)} \zeta_{* 2}\left(\frac{5}{2}-\left|\zeta_{*}\right|^{2}\right) e^{-\frac{1}{2}\left|\zeta_{*}\right|^{2}} d \zeta_{*} \\
=: & -A_{-}+B_{-}+A_{+}-B_{+},
\end{aligned}
$$

where $\nu_{*}=\nu\left(\left|\zeta_{*}\right|\right)$. Introducing new variables $\rho, \alpha, \varphi$ that represent $\zeta_{*}$ by

$$
\zeta_{*}=\left(\rho \alpha, \rho \sqrt{1-\alpha^{2}} \cos \varphi, \rho \sqrt{1-\alpha^{2}} \sin \varphi\right),
$$

where $\rho \in[0, \infty), \alpha \in[-1,1]$, and $\varphi \in[0,2 \pi)$, we can write

$$
\begin{aligned}
& A_{ \pm}=\int_{0}^{1} \int_{0}^{2 \pi} \int_{0}^{\infty} g_{\zeta}(\rho, \pm \alpha, \varphi) \frac{1}{\alpha} e^{-\frac{\nu(\rho)}{\kappa \rho \alpha}\left(\frac{1}{2} \pm s\right)} d \rho d \varphi d \alpha, \\
& B_{ \pm}=e^{-\frac{|\zeta|^{2}}{2}} \int_{0}^{1} \int_{0}^{2 \pi} \int_{0}^{\infty} h_{\zeta}(\rho, \pm \alpha, \varphi) \frac{1}{\alpha} e^{-\frac{\nu(\rho)}{\kappa \rho \alpha}\left(\frac{1}{2} \pm s\right)} d \rho d \varphi d \alpha .
\end{aligned}
$$

Lemma 3.1 is a consequence of the following estimates for $A_{ \pm}$and $B_{ \pm}$:

$$
\begin{aligned}
A_{ \pm}= & -\ln \left(\frac{\frac{1}{2} \pm s}{\kappa}\right)\left(\int_{0}^{2 \pi} \int_{0}^{\infty} g_{\zeta}(\rho, 0, \varphi) d \rho d \varphi+O\left(\frac{1}{\kappa^{2}}\right)\right)+O(1) \\
B_{ \pm}= & -\ln \left(\frac{\frac{1}{2} \pm s}{\kappa}\right)\left(e^{-\frac{|\zeta|^{2}}{2}} \int_{0}^{2 \pi} \int_{0}^{\infty} h_{\zeta}(\rho, 0, \varphi) d \rho d \varphi+O\left(\frac{1}{\kappa^{2}}\right) e^{-\frac{|\zeta|^{2}}{4}}\right) \\
& +O(1) e^{-\frac{|\zeta|^{2}}{4}}
\end{aligned}
$$

The estimates for $A_{+}$and $B_{+}$can be obtained from those of $A_{-}$and $B_{-}$by replacing $\frac{1}{2}-s$ and $-\zeta_{1}$ with $\frac{1}{2}+s$ and $\zeta_{1}$, and the estimate for $B_{-}$is similar and in fact simpler than that for $A_{-}$. Thus, we will only prove the estimate for $A_{-}$.

From (1.7),

$$
\frac{d}{d \rho}\left(\frac{\nu(\rho)}{\rho}\right)=-\frac{1}{\sqrt{2} \rho^{3}} \int_{0}^{\rho} e^{-s^{2}} d s<0 .
$$


Thus, $\frac{\nu(\rho)}{\rho}$ is a monotone decreasing function of $\rho$. From the well-known bounds (1.10), we have $\nu_{1}\left(1+\frac{1}{\rho}\right) \geq \frac{\nu(\rho)}{\rho} \geq \nu_{0}\left(1+\frac{1}{\rho}\right)$. Therefore, $\frac{\nu(\rho)}{\rho}$ tends to infinity as $\rho \rightarrow 0$ and converges to some positive constant as $\rho \rightarrow \infty$. Thus, for $\kappa>2 \nu_{1}$, there exists a unique solution $\rho=\rho_{s}$ to $\frac{\nu(\rho)}{\kappa \rho}\left(\frac{1}{2}-s\right)=1$. We use $\rho_{s}$ to divide the integration in $A_{-}$into two, $A_{-}=A_{1}+A_{2}$.

Consider first

$$
A_{1}:=\int_{0}^{\rho_{s}} \int_{0}^{2 \pi} \int_{0}^{1} g_{\zeta}(\rho,-\alpha, \varphi) \frac{1}{\alpha} e^{-\frac{\nu(\rho)}{\kappa \rho \alpha}\left(\frac{1}{2}-s\right)} d \alpha d \varphi d \rho .
$$

Note that

$$
\left|\frac{1}{\alpha} e^{-\frac{\nu(\rho)}{\kappa \rho \alpha}\left(\frac{1}{2}-s\right)}\right|=\left|\frac{\nu(\rho)}{\kappa \rho \alpha}\left(\frac{1}{2}-s\right) e^{-\frac{\nu(\rho)}{\kappa \rho \alpha}\left(\frac{1}{2}-s\right)}\right| \cdot\left|\frac{\kappa \rho}{\nu(\rho)\left(\frac{1}{2}-s\right)}\right|<C\left|\frac{\kappa \rho}{\nu(\rho)\left(\frac{1}{2}-s\right)}\right| .
$$

Using this and applying Hölder's inequality, we have

$$
\begin{aligned}
\left|A_{1}\right| \leq & C\left(\int_{0}^{\rho_{s}} \int_{0}^{2 \pi} \int_{0}^{1}\left|\zeta-\zeta_{*}\right|^{-2} e^{-\frac{\left(|\zeta|^{2}-\rho^{2}\right)^{2}}{2\left|\zeta-\zeta_{*}\right|^{2}}-\frac{1}{2}\left|\zeta-\zeta_{*}\right|^{2}}\left|\frac{5}{2}-\rho^{2}\right| e^{-\frac{\rho^{2}}{2}} \rho^{2} d \rho d \varphi d \alpha\right)^{\frac{1}{2}} \\
& \cdot\left(\int_{0}^{\rho_{s}} \int_{0}^{2 \pi} \int_{0}^{1}\left|\frac{5}{2}-\rho^{2}\right| e^{-\frac{\rho^{2}}{2}} \rho^{2}\left|\frac{\kappa \rho}{\nu(\rho)\left(\frac{1}{2}-s\right)}\right|^{2} d \alpha d \varphi d \rho\right)^{\frac{1}{2}} .
\end{aligned}
$$

The first term on the right-hand side is finite. Since $\left|\frac{\kappa \rho}{\nu(\rho)\left(\frac{1}{2}-s\right)}\right| \leq 1$ in the domain of integration, the second term is also finite. Thus, we obtain

$$
\left|A_{1}\right|=O(1)
$$

Next, consider $A_{2}$ :

$$
\begin{aligned}
A_{2} & :=\int_{0}^{2 \pi} \int_{\rho_{s}}^{\infty} \int_{0}^{1} g_{\zeta}(\rho,-\alpha, \varphi) \frac{1}{\alpha} e^{-\frac{\nu(\rho)}{\kappa \rho} \frac{(1-s)}{\alpha}} d \alpha d \rho d \varphi \\
& =\int_{0}^{2 \pi} \int_{\rho_{s}}^{\infty} \int_{0}^{1} g_{\zeta}(\rho,-\alpha, \varphi) \frac{\partial}{\partial \alpha}\left(\int_{1}^{\alpha} \frac{1}{z} e^{-\frac{\nu(\rho)}{\kappa \rho} \frac{\left(1 \frac{1}{2}-s\right)}{z}} d z\right) d \alpha d \rho d \varphi .
\end{aligned}
$$

Introducing exponential integral

$$
E_{1}(x)=\int_{1}^{\infty} e^{-t x} t^{-1} d t=\int_{x}^{\infty} e^{-s} s^{-1} d s
$$


we have

$$
\int_{1}^{\alpha} \frac{1}{z} e^{-\frac{\nu(\rho)}{\kappa \rho}\left(\frac{1}{2}-s\right)} d z=E_{1}\left(\frac{\nu(\rho)\left(\frac{1}{2}-s\right)}{\kappa \rho \alpha}\right)-E_{1}\left(\frac{\nu(\rho)\left(\frac{1}{2}-s\right)}{\kappa \rho}\right) .
$$

There is a well-known expansion for $E_{1}$ (for example see [1]),

$$
E_{1}(x)=-\gamma-\ln x+\sum_{k=1}^{\infty} \frac{(-1)^{k+1} x^{k}}{k \cdot k !} .
$$

We also have

$$
\frac{1}{2} e^{-x} \ln \left(1+\frac{2}{x}\right) \leq E_{1}(x) \leq e^{-x} \ln \left(1+\frac{1}{x}\right) \text { for } x>0 .
$$

Noting that

$$
\frac{\nu(\rho)}{\kappa \rho} \frac{\left(\frac{1}{2}-s\right)}{\alpha}>\frac{\nu_{0}\left(\frac{1}{2}-s\right)}{\kappa \alpha},
$$

we see, for fixed $s$ and $\kappa$, that $E_{1}\left(\frac{\nu(\rho)\left(\frac{1}{2}-s\right)}{\kappa \rho \alpha}\right)$ tends to 0 uniformly with respect to $\rho$ as $\alpha$ tends to zero. Using integration by parts, we obtain

$$
\begin{aligned}
A_{2}= & \int_{0}^{2 \pi} \int_{\rho_{s}}^{\infty} g_{\zeta}(\rho, 0, \varphi) E_{1}\left(\frac{\nu(\rho)\left(\frac{1}{2}-s\right)}{\kappa \rho}\right) d \rho d \varphi \\
& +\int_{0}^{2 \pi} \int_{\rho_{s}}^{\infty} \int_{0}^{1}\left(E_{1}\left(\frac{\nu(\rho)\left(\frac{1}{2}-s\right)}{\kappa \rho}\right)-E_{1}\left(\frac{\nu(\rho)\left(\frac{1}{2}-s\right)}{\kappa \rho \alpha}\right)\right) \\
& \cdot \frac{\partial}{\partial \alpha} g_{\zeta}(\rho,-\alpha, \varphi) d \alpha d \rho d \varphi .
\end{aligned}
$$

Since (3.15) implies

$$
\left|E_{1}\left(\frac{\nu(\rho)\left(\frac{1}{2}-s\right)}{\kappa \rho}\right)+\ln \left(\frac{\frac{1}{2}-s}{\kappa}\right)+\ln \left(\frac{\nu(\rho)}{\rho}\right)\right|<C, \quad \text { for } \rho_{s} \leq \rho<\infty,
$$

we can rewrite the first term on the right-hand side and obtain

$$
\begin{aligned}
A_{2}= & -\ln \left(\frac{\frac{1}{2}-s}{\kappa}\right) \int_{0}^{2 \pi} \int_{\rho_{s}}^{\infty} g_{\zeta}(\rho, 0, \varphi) d \rho d \varphi \\
& +\int_{0}^{2 \pi} \int_{\rho_{s}}^{\infty} g_{\zeta}(\rho, 0, \varphi) O(1) d \rho d \varphi-\int_{0}^{2 \pi} \int_{\rho_{s}}^{\infty} \ln \left(\frac{\nu(\rho)}{\rho}\right) g_{\zeta}(\rho, 0, \varphi) d \rho d \varphi \\
& -\int_{0}^{2 \pi} \int_{\rho_{s}}^{\infty} \int_{0}^{1}\left(E_{1}\left(\frac{\nu(\rho)\left(\frac{1}{2}-s\right)}{\kappa \rho \alpha}\right)-E_{1}\left(\frac{\nu(\rho)\left(\frac{1}{2}-s\right)}{\kappa \rho}\right)\right) \\
& \cdot \frac{\partial}{\partial \alpha} g_{\zeta}(\rho,-\alpha, \varphi) d \alpha d \rho d \varphi \\
= & : A_{2}^{\prime}+R_{1}+R_{2}+R_{3}+R_{4} .
\end{aligned}
$$


Note that the singularity of $A_{2}^{\prime}$ is explicit:

$$
A_{2}^{\prime}=-\ln \left(\frac{\frac{1}{2}-s}{\kappa}\right) \int_{0}^{2 \pi} \int_{\rho_{s}}^{\infty} g_{\zeta}(\rho, 0, \varphi) d \rho d \varphi .
$$

Below we first prove that

$$
A_{2}^{\prime}=-\ln \left(\frac{\frac{1}{2}-s}{\kappa}\right)\left[\int_{0}^{2 \pi} \int_{0}^{\infty} g_{\zeta}(\rho, 0, \varphi) d \rho d \varphi+O\left(\frac{1}{\kappa^{2}}\right)\right]
$$

by showing that

$$
\int_{0}^{2 \pi} \int_{0}^{\rho_{s}} g_{\zeta}(\rho, 0, \varphi) d \rho d \varphi=O\left(\frac{1}{\kappa^{2}}\right)
$$

Then we prove that $R_{1}, R_{2}, R_{3}$, and $R_{4}$ are $O(1)$ and conclude the estimate (3.8) for $A_{-}$.

For the estimate of $A_{2}^{\prime}$, we first estimate $\rho_{s}$. Recalling the inequality (1.10), we see from the definition of $\rho_{s}$ that

$$
\rho_{s}<\frac{\nu_{1}\left(\frac{1}{2}-s\right)}{\kappa}\left(1+\rho_{s}\right)
$$

Since $\kappa>2 \nu_{1}$, we have

$$
\rho_{s}<\frac{2 \nu_{1}\left(\frac{1}{2}-s\right)}{\kappa}<1, \rho_{s}=O(1) \frac{1}{\kappa}
$$

Therefore, we can prove (3.20) by showing that

$$
\left|\int_{0}^{2 \pi} \int_{0}^{\rho_{s}} g_{\zeta}(\rho, 0, \varphi) d \rho d \varphi\right| \leq C \rho_{s}^{2}
$$

Because $\rho_{s}<1$, inside the domain of integration we have

$$
\left|g_{\zeta}(\rho, 0, \varphi)\right| \leq \frac{C \rho^{2}}{\sqrt{\left(\zeta_{2}-\rho \cos \varphi\right)^{2}+\left(\zeta_{3}-\rho \sin \varphi\right)^{2}}} .
$$

When $\left(\zeta_{2}^{2}+\zeta_{3}^{2}\right)^{\frac{1}{2}}>2 \rho_{s}$, inside the domain of integration we have

$$
\sqrt{\left(\zeta_{2}-\rho \cos \varphi\right)^{2}+\left(\zeta_{3}-\rho \sin \varphi\right)^{2}}>\left(\zeta_{2}^{2}+\zeta_{3}^{2}\right)^{\frac{1}{2}}-\rho>\rho_{s}>\rho,
$$


and thus

$$
\left|\int_{0}^{2 \pi} \int_{0}^{\rho_{s}} g_{\zeta}(\rho, 0, \phi) d \rho d \varphi\right| \leq C \int_{0}^{2 \pi} \int_{0}^{\rho_{s}} \rho d \rho d \varphi \leq \pi C \rho_{s}^{2} .
$$

When $\left(\zeta_{2}^{2}+\zeta_{3}^{2}\right)^{\frac{1}{2}} \leq 2 \rho_{s}$, we change the variables by the following relation

$$
\begin{aligned}
& r \cos \theta=\zeta_{2}-\rho \cos \varphi \\
& r \sin \theta=\zeta_{3}-\rho \sin \varphi
\end{aligned}
$$

Since the Jacobian is $r / \rho$, inside the domain of integration we have

$$
r=\sqrt{\left(\zeta_{2}-\rho \cos \varphi\right)^{2}+\left(\zeta_{3}-\rho \sin \varphi\right)^{2}} \leq\left(\zeta_{2}^{2}+\zeta_{3}^{2}\right)^{\frac{1}{2}}+\rho \leq 3 \rho_{s}
$$

and thus

$$
\left|\int_{0}^{2 \pi} \int_{0}^{\rho_{s}} g_{\zeta}(\rho, 0, \varphi) d \rho d \varphi\right| \leq C \int_{0}^{2 \pi} \int_{0}^{3 \rho_{s}} \rho_{s} d r d \theta \leq 6 \pi C \rho_{s}^{2}
$$

Combining (3.22) and (3.23) proves (3.21) and (3.20), and thus the estimate (3.19) for $A_{2}^{\prime}$ is obtained.

Next we estimate $R_{1}$ and $R_{2}$. Estimate for $R_{1}$ is readily obtained as

$$
\left|R_{1}\right|=O(1) \int_{0}^{\infty} \int_{0}^{2 \pi}\left|\zeta-\zeta_{*}\right|^{-1} e^{-\frac{|\zeta-\zeta *|^{2}}{4}-\frac{\rho^{2}}{4}} \rho d \varphi d \rho=O(1)
$$

For $R_{2}$, we divide $R_{2}$ into $R_{2 l}$ and $R_{2 s}$ whose domains of integration are $\rho>1$ and $\rho_{s}<\rho<1$ respectively. $R_{2 l}$ is bounded since $\nu_{0}<\frac{\nu(\rho)}{\rho}<2 \nu_{1}$ by (1.10) when $\rho>1$. Since $\nu_{0}<\nu(\rho)<2 \nu_{1}$ when $\rho<1$, we have

$$
\begin{aligned}
& \left|R_{2 s}\right| \leq C \int_{\rho_{s}}^{1} \int_{0}^{2 \pi}|\ln \rho|\left|g_{\zeta}(\rho, 0, \varphi)\right| d \varphi d \rho \\
& \leq C\left(\int_{\rho_{s}}^{1} \int_{0}^{2 \pi}|\ln \rho|^{q} \rho d \varphi d \rho\right)^{\frac{1}{q}}\left(\int_{\rho_{s}}^{1} \int_{0}^{2 \pi}\left|\zeta-\zeta_{*}\right|^{-p} \rho d \varphi d \rho\right)^{\frac{1}{p}} \leq C,
\end{aligned}
$$

provided $1<p<2$ and $q$ is the Hölder's conjugate of $p$. Thus $R_{2}$ is also $O(1)$. 
To finish the proof of (3.8) for $A_{-}$, it remains to show that $R_{3}+R_{4}=O(1)$.

For this, we first study the $L_{1}$ norm of $\frac{\partial}{\partial \alpha} g_{\zeta}$. By direct calculation,

$$
\begin{aligned}
\frac{1}{c_{1}} \frac{\partial g_{\zeta}}{\partial \alpha}= & e^{-\frac{\left(|\zeta|^{2}-\rho^{2}\right)^{2}}{4\left|\zeta-\zeta_{*}\right|^{2}}-\frac{1}{4}\left|\zeta-\zeta_{*}\right|^{2}} \cos \varphi\left(\frac{5}{2}-\rho^{2}\right) e^{-\frac{\rho^{2}}{2}} \rho^{2}\left(-\frac{1}{\left|\zeta-\zeta_{*}\right|} \frac{\alpha}{\sqrt{1-\alpha^{2}}}\right. \\
& \left.+\sqrt{1-\alpha^{2}} \frac{\partial\left|\zeta-\zeta_{*}\right|}{\partial \alpha}\left(-\frac{1}{\left|\zeta-\zeta_{*}\right|^{2}}+\frac{\left(|\zeta|^{2}-\rho^{2}\right)^{2}}{2\left|\zeta-\zeta_{*}\right|^{4}}-\frac{1}{2}\right)\right)
\end{aligned}
$$

where

$$
\frac{\partial\left|\zeta-\zeta_{*}\right|}{\partial \alpha}=\frac{1}{\left|\zeta-\zeta_{*}\right|}\left(\zeta_{1} \rho+\zeta_{2} \rho \cos \varphi \frac{\alpha}{\sqrt{1-\alpha^{2}}}+\zeta_{3} \rho \sin \varphi \frac{\alpha}{\sqrt{1-\alpha^{2}}}\right)
$$

To estimate $\frac{\partial\left|\zeta-\zeta_{*}\right|}{\partial \alpha}$, we let

$$
T=\left(\rho \sqrt{1-\alpha^{2}}, \rho \alpha \cos \varphi, \rho \alpha \sin \varphi\right)
$$

Note that $\zeta_{*} \cdot T=0$; thus

$$
\left(\zeta_{1} \rho+\zeta_{2} \rho \cos \varphi \frac{\alpha}{\sqrt{1-\alpha^{2}}}+\zeta_{3} \rho \sin \varphi \frac{\alpha}{\sqrt{1-\alpha^{2}}}\right)=\frac{\left(\zeta-\zeta_{*}\right) \cdot T}{\sqrt{1-\alpha^{2}}} .
$$

Therefore,

$$
\left|\sqrt{1-\alpha^{2}} \frac{\partial\left|\zeta-\zeta_{*}\right|}{\partial \alpha}\right|=\left|\frac{\left(\zeta-\zeta_{*}\right) \cdot T}{\left|\zeta-\zeta_{*}\right|}\right| \leq|T|=\rho .
$$

Applying the triangular inequality, we have

$$
\frac{\left(|\zeta|^{2}-\rho^{2}\right)^{2}}{\left|\zeta-\zeta_{*}\right|^{4}} \leq \frac{(|\zeta|+\rho)^{2}}{\left|\zeta-\zeta_{*}\right|^{2}} \leq 2+\frac{8 \rho^{2}}{\left|\zeta-\zeta_{*}\right|^{2}}
$$

Using both (3.25) and (3.24), we can conclude

$$
\left|\frac{\partial g_{\zeta}}{\partial \alpha}\right| \leq C e^{-\frac{\rho^{2}}{4}-\frac{\left|\zeta-\zeta_{*}\right|^{2}}{4}} \rho^{2}\left(\frac{1}{\left|\zeta-\zeta_{*}\right|} \frac{\alpha}{\sqrt{1-\alpha^{2}}}+\frac{1}{\left|\zeta-\zeta_{*}\right|^{2}}+1\right)
$$

On one hand, since the integration is in the three dimensional space, we have

$$
\int_{0}^{2 \pi} \int_{0}^{\infty} \int_{0}^{1} e^{-\frac{\rho^{2}}{4}-\frac{|\zeta-\zeta *|^{2}}{4}} \rho^{2}\left(\mid \frac{1}{\left|\zeta-\zeta_{*}\right|^{2}}+1\right) d \alpha d \rho d \varphi<C .
$$


On the other hand, by the Hölder's inequality, we have

$$
\begin{aligned}
& \int_{0}^{2 \pi} \int_{0}^{\infty} \int_{0}^{1}\left|e^{-\frac{\rho^{2}}{4}-\frac{\left|\zeta-\zeta_{*}\right|^{2}}{4}} \rho^{2} \frac{1}{\left|\zeta-\zeta_{*}\right|} \frac{\alpha}{\sqrt{1-\alpha^{2}}}\right| d \alpha d \rho d \varphi \\
\leq & \left(\iiint\left|e^{-\frac{\rho^{2}}{4}-\frac{\left|\zeta-\zeta_{*}\right|^{2}}{4}} \rho^{2} \frac{1}{\left|\zeta-\zeta_{*}\right|^{p}}\right| d \alpha d \rho d \varphi\right)^{\frac{1}{p}} \\
& \cdot\left(\iiint\left|e^{-\frac{\rho^{2}}{4}-\frac{\left|\zeta-\zeta_{*}\right|^{2}}{4}} \rho^{2} \frac{\alpha^{q}}{\sqrt{1-\alpha^{2}}}\right| d \alpha d \rho d \varphi\right)^{\frac{1}{q}},
\end{aligned}
$$

where $p=\frac{12}{5}<3, q=\frac{12}{7}<2$. Therefore, we obtain

$$
\int_{0}^{2 \pi} \int_{0}^{\infty} \int_{0}^{1}\left|\frac{\partial g_{\zeta}(\rho,-\alpha, \varphi)}{\partial \alpha}\right| d \alpha d \rho d \varphi<C .
$$

With the above estimate $(3.27)$ for $L_{1}$ norm of $\frac{\partial}{\partial \alpha} g_{\zeta}$, we estimate $R_{3}+R_{4}$. We divide the domain of integration into two parts:

$$
\begin{aligned}
& D_{H}=\left\{(\rho, \alpha, \varphi) \mid \rho \in\left(\rho_{s}, \infty\right), \alpha \in\left(0, \frac{\nu(\rho)\left(\frac{1}{2}-s\right)}{\kappa \rho}\right), \varphi \in(0,2 \pi)\right\}, \\
& D_{M}=\left\{(\rho, \alpha, \varphi) \mid \rho \in\left(\rho_{s}, \infty\right), \alpha \in\left(\frac{\nu(\rho)\left(\frac{1}{2}-s\right)}{\kappa \rho}, 1\right), \varphi \in(0,2 \pi)\right\},
\end{aligned}
$$

and denote the individual parts of $R_{3}$ and $R_{4}$ by putting the corresponding suffix as $R_{3 H}, R_{4 H}, R_{3 M}$, and $R_{4 M}$. In $D_{H}$, we have $\frac{\nu(\rho)\left(\frac{1}{2}-s\right)}{\kappa \rho \alpha}>1$. Thus, applying (3.16), we have $E_{1}\left(\frac{\nu(\rho)\left(\frac{1}{2}-s\right)}{\kappa \rho \alpha}\right)<C$. Therefore,

$$
\left|R_{3 H}\right| \leq C
$$

as a consequence of (3.27). For $R_{4 H}$, because of (3.17), we only need to estimate the following integral:

$$
\int_{\rho_{s}}^{\infty} \int_{0}^{2 \pi} \int_{0}^{\frac{\nu(\rho)\left(\frac{1}{2}-s\right)}{\kappa \rho}} \ln \left(\frac{\nu(\rho)\left(\frac{1}{2}-s\right)}{\kappa \rho}\right) \frac{\partial}{\partial \alpha} g_{\zeta} d \alpha d \varphi d \rho .
$$

Using (3.26), the task reduces to estimating the following integrals:

$$
\begin{aligned}
& R_{4 H}^{\prime}:=\int_{D_{H}} \ln \left(\frac{\nu(\rho)\left(\frac{1}{2}-s\right)}{\kappa \rho}\right) e^{-\frac{\rho^{2}}{4}-\frac{\left|\zeta-\zeta_{*}\right|^{2}}{4}} \rho^{2} \frac{1}{\left|\zeta-\zeta_{*}\right|} \frac{\alpha}{\sqrt{1-\alpha^{2}}} d \alpha d \varphi d \rho, \\
& R_{4 H}^{\prime \prime}:=\int_{D_{H}} \ln \left(\frac{\nu(\rho)\left(\frac{1}{2}-s\right)}{\kappa \rho}\right) e^{-\frac{\rho^{2}}{4}-\frac{\left|\zeta-\zeta_{*}\right|^{2}}{4}} \rho^{2}\left(\frac{1}{\left|\zeta-\zeta_{*}\right|^{2}}+1\right) d \alpha d \varphi d \rho
\end{aligned}
$$


Again by the Hölder's inequality, we have

$$
\begin{aligned}
\left|R_{4 H}^{\prime}\right| \leq & \left(\int_{D_{H}} e^{-\frac{\rho^{2}}{4}-\frac{\left|\zeta-\zeta_{*}\right|^{2}}{4}} \rho^{2}\left(\frac{1}{\left|\zeta-\zeta_{*}\right|}\right)^{a} d \alpha d \varphi d \rho\right)^{\frac{1}{a}} \\
& \cdot\left(\int_{D_{H}} e^{-\frac{\rho^{2}}{4}-\frac{\left|\zeta-\zeta_{*}\right|^{2}}{4}} \rho^{2}\left(\frac{\alpha}{\sqrt{1-\alpha^{2}}}\right)^{b} d \alpha d \varphi d \rho\right)^{\frac{1}{b}} \\
& \cdot\left(\int_{D_{H}} e^{-\frac{\rho^{2}}{4}} \rho^{2}\left|\ln \left(\frac{\nu(\rho)\left(\frac{1}{2}-s\right)}{\kappa \rho}\right)\right|^{c}\left|\frac{\nu(\rho)\left(\frac{1}{2}-s\right)}{\kappa \rho}\right| d \alpha d \varphi d \rho\right)^{\frac{1}{c}}<C,
\end{aligned}
$$

and

$$
\begin{aligned}
\left|R_{4 H}^{\prime \prime}\right| \leq & \left(\int_{D_{H}} e^{-\frac{\rho^{2}}{4}-\frac{\left|\zeta-\zeta_{*}\right|^{2}}{4}} \rho^{2}\left(\frac{1}{\left|\zeta-\zeta_{*}\right|^{2}}+1\right)^{p} d \alpha d \varphi d \rho\right)^{\frac{1}{p}} \\
& \cdot\left(\int_{D_{H}} e^{-\frac{\rho^{2}}{4}} \rho^{2}\left|\ln \left(\frac{\nu(\rho)\left(\frac{1}{2}-s\right)}{\kappa \rho}\right)\right|^{q}\left|\frac{\nu(\rho)\left(\frac{1}{2}-s\right)}{\kappa \rho}\right| d \alpha d \varphi d \rho\right)^{\frac{1}{q}} \leq C,
\end{aligned}
$$

where $a=\frac{8}{3}, b=\frac{24}{13}, c=12$ and $p=\frac{4}{3}$ and $q=4$. Therefore, we conclude

$$
\left|R_{4 H}\right|<C
$$

Finally we deal with $R_{3 M}$ and $R_{4 M}$ together. In $D_{M}$ we have $\frac{\nu(\rho)\left(\frac{1}{2}-s\right)}{\kappa \rho \alpha}<1$ and thus from (3.15)

$$
\left|E_{1}\left(\frac{\nu(\rho)\left(\frac{1}{2}-s\right)}{\kappa \rho \alpha}\right)+\ln \left(\frac{\nu(\rho)\left(\frac{1}{2}-s\right)}{\kappa \rho \alpha}\right)\right| \leq C .
$$

Combining with (3.17), we have

$$
\left|E_{1}\left(\frac{\nu(\rho)\left(\frac{1}{2}-s\right)}{\kappa \rho}\right)-E_{1}\left(\frac{\nu(\rho)\left(\frac{1}{2}-s\right)}{\kappa \rho \alpha}\right)+\ln \alpha\right| \leq C .
$$

Thus, again using (3.26) and (3.31), we only need to estimate the following integrals:

$$
\begin{aligned}
\left(R_{3 M}+R_{4 M}\right)^{\prime} & :=\int_{D_{M}} e^{-\frac{\rho^{2}}{4}-\frac{\left|\zeta-\zeta_{*}\right|^{2}}{4}} \rho^{2} \frac{1}{\left|\zeta-\zeta_{*}\right|} \frac{\alpha}{\sqrt{1-\alpha^{2}}} \ln \alpha d \alpha d \rho d \varphi \\
\left(R_{3 M}+R_{4 M}\right)^{\prime \prime} & :=\int_{D_{M}} e^{-\frac{\rho^{2}}{4}-\frac{\left|\zeta-\zeta_{*}\right|^{2}}{4}} \rho^{2}\left(\frac{1}{\left|\zeta-\zeta_{*}\right|^{2}}+1\right) \ln \alpha d \alpha d \rho d \varphi
\end{aligned}
$$


For both estimates, we apply the Hölder's inequality:

$$
\begin{aligned}
\left|\left(R_{3 M}+R_{4 M}\right)^{\prime}\right| \leq & \left(\int_{D_{M}} e^{-\frac{\rho^{2}}{4}-\frac{\left|\zeta-\zeta_{*}\right|^{2}}{4}} \rho^{2}\left(\frac{1}{\left|\zeta-\zeta_{*}\right|}\right)^{a} d \alpha d \rho d \varphi\right)^{\frac{1}{a}} \\
& \cdot\left(\int_{D_{M}} e^{-\frac{\rho^{2}}{4}-\frac{\left|\zeta-\zeta_{*}\right|^{2}}{4}} \rho^{2}\left(\frac{\alpha}{\sqrt{1-\alpha^{2}}}\right)^{b} d \alpha d \rho d \varphi\right)^{\frac{1}{b}} \\
& \cdot\left(\int_{D_{M}} e^{-\frac{\rho^{2}}{4}-\frac{\left|\zeta-\zeta_{*}\right|^{2}}{4}} \rho^{2}|\ln \alpha|^{c} d \alpha d \rho d \varphi\right)^{\frac{1}{c}}<C, \\
\left|\left(R_{3 M}+R_{4 M}\right)^{\prime \prime}\right| \leq & \left(\int_{D_{M}} e^{-\frac{\rho^{2}}{4}-\frac{\left|\zeta-\zeta_{*}\right|^{2}}{4}} \rho^{2}\left(\frac{1}{\left|\zeta-\zeta_{*}\right|^{2}}+1\right)^{p} d \alpha d \rho d \varphi\right)^{\frac{1}{p}} \\
& \cdot\left(\int_{D_{M}} e^{-\frac{\rho^{2}}{4}-\frac{\left|\zeta-\zeta_{*}\right|^{2}}{4}} \rho^{2}|\ln \alpha|^{q} d \alpha d \rho d \varphi\right)^{\frac{1}{q}}<C,
\end{aligned}
$$

where we choose $a=\frac{8}{3}<3, b=\frac{24}{13}<2, c=12$ and $p=\frac{4}{3}<\frac{3}{2}, q=4$. Combining (3.32) and (3.33), we conclude

$$
\left|R_{3 M}+R_{4 M}\right| \leq C
$$

This is the end of the proof of (3.8) for $A_{-}$, and we complete the proof of Lemma 3.1.

\section{Further studies on $F$}

In this section, we will investigate the dependence of variables for $F$, namely $F(\zeta)=\zeta_{2} \bar{F}\left(\zeta_{1}, \zeta_{r}\right)$, where $\zeta_{r}=\left(\zeta_{2}^{2}+\zeta_{3}^{2}\right)^{\frac{1}{2}}$, to conclude Lemma 2.2 from Lemma 3.1. We will further study $\bar{F}$, estimate it near $\zeta_{1}=0$, and prove Lemma 2.3.

\section{Definition 1.}

$$
\bar{F}\left(\zeta_{1}, \zeta_{r}\right)=-G\left(\zeta_{1}, \zeta_{r}\right)+H\left(\zeta_{1}, \zeta_{r}\right)
$$

where

$$
\begin{aligned}
G\left(\zeta_{1}, \zeta_{r}\right)=e^{-\frac{\zeta_{1}^{2}+\zeta_{r}^{2}}{2}} \int_{0}^{2 \pi} \int_{0}^{\infty} \frac{c_{1} P}{\sqrt{\zeta_{1}^{2}+P^{2}}} e^{-\frac{P^{2}\left(P+\zeta_{r} \cos \beta\right)^{2}}{\zeta_{1}^{2}+P^{2}}} \\
\cdot\left(\frac{5}{2}-P^{2}-\zeta_{r}^{2}-2 \zeta_{r} P \cos \beta\right)\left(1+\frac{P}{\zeta_{r}} \cos \beta\right) d P d \beta,
\end{aligned}
$$




$$
\begin{aligned}
H\left(\zeta_{1}, \zeta_{r}\right)=e^{-\frac{\zeta_{1}^{2}+\zeta_{r}^{2}}{2}} \int_{0}^{2 \pi} \int_{0}^{\infty} c_{2} P \sqrt{\zeta_{1}^{2}+P^{2}}\left(1+\frac{P \cos \beta}{\zeta_{r}}\right) \\
\cdot\left(\frac{5}{2}-P^{2}-\zeta_{r}^{2}-2 \zeta_{r} P \cos \beta\right) e^{-P^{2}-\zeta_{r}^{2}-2 \zeta_{r} P \cos \beta} d P d \beta
\end{aligned}
$$

The following lemma, combined with Lemma 3.1, immediately concludes Lemma 2.2.

\section{Lemma 4.1.}

$$
F\left(\zeta_{1}, \zeta_{2}, \zeta_{3}\right)=\zeta_{2} \bar{F}\left(\zeta_{1}, \zeta_{r}\right)
$$

Proof. In (3.2), since $\alpha=0$, the variables $(\rho, \varphi)$ can be viewed as the polar coordinates for two dimensional vector $\left(\zeta_{* 2}, \zeta_{* 3}\right): \zeta_{* 2}=\rho \cos \varphi$ and $\zeta_{* 3}=\rho \sin \varphi$. We introduce another polar coordinates $(P, \beta)$ centered at $\left(\zeta_{2}, \zeta_{3}\right)$ with its north pole in the direction $e=\left(\frac{\zeta_{2}}{\zeta_{r}}, \frac{\zeta_{3}}{\zeta_{r}}\right)$ for the same vector:

$$
\begin{aligned}
& \zeta_{* 2}(=\rho \cos \varphi)=\zeta_{2}+P \cos \beta \frac{\zeta_{2}}{\zeta_{r}}+P \sin \beta \frac{\zeta_{3}}{\zeta_{r}} \\
& \zeta_{* 3}(=\rho \sin \varphi)=\zeta_{3}-P \cos \beta \frac{\zeta_{3}}{\zeta_{r}}+P \sin \beta \frac{\zeta_{2}}{\zeta_{r}}
\end{aligned}
$$

Changing the variables from $\rho$ and $\varphi$ to $P$ and $\beta$ in (3.2), we obtain

$$
\begin{aligned}
& F=-\int_{0}^{2 \pi} \int_{0}^{\infty} \frac{c_{1} P}{\sqrt{\zeta_{1}^{2}+P^{2}}}\left(\zeta_{2}+P \frac{\zeta_{2}}{\zeta_{r}} \cos \beta\right. \\
&\left.+P \sin \beta \frac{\zeta_{3}}{\zeta_{r}}\right)\left(\frac{5}{2}-\rho^{2}\right) e^{-\frac{\left(\zeta_{1}^{2}+\zeta_{r}^{2}-\rho^{2}\right)^{2}}{4\left(\zeta_{1}^{2}+P^{2}\right)}-\frac{\zeta_{1}^{2}+P^{2}}{4}-\frac{\rho^{2}}{2}} d P d \beta \\
&+e^{-\frac{\zeta_{1}^{2}+\zeta_{r}^{2}}{2}} \int_{0}^{2 \pi} \int_{0}^{\infty} c_{2} P \sqrt{\zeta_{1}^{2}+P^{2}}\left(\zeta_{2}+P \cos \beta \frac{\zeta_{2}}{\zeta_{r}}\right. \\
&\left.+P \sin \beta \frac{\zeta_{3}}{\zeta_{r}}\right)\left(\frac{5}{2}-\rho^{2}\right) e^{-\rho^{2}} d P d \beta,
\end{aligned}
$$

where

$$
\rho^{2}=\zeta_{r}^{2}+P^{2}+2 \zeta_{r} P \cos \beta
$$

Since $\sin \beta$ is antisymmetric with respect to $\beta=\pi$, the integral of terms with $\zeta_{3}$ vanishes. By substituting $\rho^{2}=\zeta_{r}^{2}+P^{2}+2 \zeta_{r} P \cos \beta$, after a straightforward calculation, we obtain (4.4). 
In viewing (4.2) and (4.3), the integrand is singular at $\zeta_{r}=0$ in the integrand in both cases. However, it is removable in the following way. We rewrite (4.2) and (4.3) as

$$
\begin{aligned}
& G\left(\zeta_{1}, \zeta_{r}\right)=\int_{0}^{2 \pi} \int_{0}^{\infty} \frac{c_{1} P}{\sqrt{\zeta_{1}^{2}+P^{2}}}\left(1+\frac{P}{\zeta_{r}} \cos \beta\right) e^{-\frac{\zeta_{1}^{2}}{2}-\frac{P^{4}}{\zeta_{1}^{2}+P^{2}}} g\left(\zeta_{1}, \zeta_{r}, P, \beta\right) d P d \beta, \\
& H\left(\zeta_{1}, \zeta_{r}\right)=\int_{0}^{2 \pi} \int_{0}^{\infty} c_{2} P \sqrt{\zeta_{1}^{2}+P^{2}}\left(1+\frac{P}{\zeta_{r}} \cos \beta\right) e^{-\frac{\zeta_{1}^{2}}{2}-P^{2}} h\left(\zeta_{r}, P, \beta\right) d P d \beta,
\end{aligned}
$$

with

$$
\begin{aligned}
g\left(\zeta_{1}, \zeta_{r}, P, \beta\right) & =\left(\frac{5}{2}-P^{2}-\zeta_{r}^{2}-2 P \zeta_{r} \cos \beta\right) e^{-\frac{\zeta_{r}^{2}}{2}-\frac{P^{2}}{\zeta_{1}^{2}+P^{2}}\left(2 \zeta_{r} P \cos \beta+\zeta_{r}^{2} \cos ^{2} \beta\right)}, \\
h\left(\zeta_{r}, P, \beta\right) & =\left(\frac{5}{2}-P^{2}-\zeta_{r}^{2}-2 \zeta_{r} P \cos \beta\right) e^{-\frac{3}{2} \zeta_{r}^{2}-2 \zeta_{r} P \cos \beta},
\end{aligned}
$$

and use the Taylor expansion of $g$ and $h$ with respect to $\zeta_{r}$ around zero to obtain

$$
\begin{aligned}
G\left(\zeta_{1}, \zeta_{r}\right)= & 2 \pi c_{1} e^{-\frac{\zeta_{1}^{2}}{2}} \int_{0}^{\infty}\left(\frac{P\left(\frac{5}{2}-2 P^{2}\right)}{\sqrt{\zeta_{1}^{2}+P^{2}}}-\frac{P^{5}\left(\frac{5}{2}-P^{2}\right)}{\sqrt{\zeta_{1}^{2}+P^{2}}}\right) e^{-\frac{P^{4}}{\zeta_{1}^{2}+P^{2}}} d P \\
& +\int_{0}^{2 \pi} \int_{0}^{\infty}\left(1+\frac{P}{\zeta_{r}} \cos \beta\right) \frac{c_{1} P}{\sqrt{\zeta_{1}^{2}+P^{2}}} e^{-\frac{\zeta_{1}^{2}}{2}-\frac{P^{4}}{\zeta_{1}^{2}+P^{2}}} R_{g} d P d \beta \\
= & : G_{1}\left(\zeta_{1}\right)+G_{2}\left(\zeta_{1}, \zeta_{r}\right), \\
H\left(\zeta_{1}, \zeta_{r}\right)= & 2 \pi c_{2} e^{-\frac{\zeta_{1}^{2}}{2}} \int_{0}^{\infty} P \sqrt{\zeta_{1}^{2}+P^{2}} e^{-P^{2}}\left(\frac{5}{2}-P^{2}-P^{2}\left(\frac{7}{2}-P^{2}\right)\right) d P \\
& +\int_{0}^{\infty} \int_{0}^{2 \pi}\left(1+\frac{P}{\zeta_{r}} \cos \beta\right) c_{2} P \sqrt{\zeta_{1}^{2}+P^{2}} e^{-\frac{\zeta_{1}^{2}}{2}-P^{2}} R_{h} d \beta d P \\
=: & H_{1}\left(\zeta_{1}\right)+H_{2}\left(\zeta_{1}, \zeta_{r}\right) .
\end{aligned}
$$


Here $R_{g}$ and $R_{h}$ are the remainder of the Taylor expansion:

$$
\begin{aligned}
R_{g}\left(\zeta_{1}, \zeta_{r}, P, \beta\right)= & g\left(\zeta_{1}, \zeta_{r}, P, \beta\right)-\left(\frac{5}{2}-P^{2}\right) \\
& +2 P \cos \beta\left(1+\frac{\left(\frac{5}{2}-P^{2}\right) P^{2}}{\zeta_{1}^{2}+P^{2}}\right) \zeta_{r} \\
R_{h}\left(\zeta_{r}, P, \beta\right)= & h\left(\zeta_{r}, P, \beta\right)-\left(\frac{5}{2}-P^{2}\right)+2 P \cos \beta\left(\frac{7}{2}-P^{2}\right) \zeta_{r} .
\end{aligned}
$$

Note that, for some $0 \leq \zeta_{r}^{\prime} \leq \zeta_{r}$ and $0 \leq \zeta_{r}^{\prime \prime} \leq \zeta_{r}$,

$$
\begin{aligned}
& R_{g}\left(\zeta_{1}, \zeta_{r}, P, \beta\right)=\frac{1}{2} \frac{\partial^{2} g}{\partial^{2} \zeta_{r}}\left(\zeta_{1}, \zeta_{r}^{\prime}, P, \beta\right) \zeta_{r}^{2}, \\
& R_{h}\left(\zeta_{r}, P, \beta\right)=\frac{1}{2} \frac{\partial^{2} h}{\partial^{2} \zeta_{r}}\left(\zeta_{r}^{\prime \prime}, P, \beta\right) \zeta_{r}^{2} .
\end{aligned}
$$

By direct calculation of these second order derivatives, we observe that both $G_{2}$ and $H_{2}$ are $O\left(\zeta_{r}\right)$; thus $G$ and $H$ are regular in $\zeta_{r}$ as well as in $\zeta_{1}$.

Next, we notice by direct calculation of (4.2) and (4.3) with $\zeta_{1}=0$ that

$$
\begin{aligned}
& G\left(0, \zeta_{r}\right)=2^{-\frac{1}{2}} \pi^{-\frac{1}{4}}\left(1-\frac{3}{8} \zeta_{r}^{2}\right) e^{-\frac{1}{2} \zeta_{r}^{2}}, \\
& H\left(0, \zeta_{r}\right)=-2^{-\frac{7}{4}} \pi^{-\frac{1}{4}} I_{0}\left(\frac{1}{2} \zeta_{r}^{2}\right) e^{-\zeta_{r}^{2}}
\end{aligned}
$$

which yields (2.4) in Lemma 2.3.

Lemma 2.4 follows the estimates below:

Lemma 4.2. For $0 \leq \bar{\zeta}_{1} \leq \zeta_{1} \leq \frac{1}{100}$,

$$
\begin{aligned}
& \left|G\left(\zeta_{1}, \zeta_{r}\right)-G\left(\bar{\zeta}_{1}, \zeta_{r}\right)\right| \leq C\left(\left|\zeta_{1}-\bar{\zeta}_{1}\right|^{\frac{1}{2}}+\left|\zeta_{1}-\bar{\zeta}_{1}\right|\right), \\
& \left|H\left(\zeta_{1}, \zeta_{r}\right)-H\left(\bar{\zeta}_{1}, \zeta_{r}\right)\right| \leq C\left|\zeta_{1}-\bar{\zeta}_{1}\right|
\end{aligned}
$$

Remark 4.1. Note that (4.17) is only of Hölder continuous type. This is due to the singularity of $k_{1}\left(\zeta, \zeta_{*}\right)$, as will be clear in the proof of the lemma.

In the rest of the present section, we prove Lemma 4.2 . 
Proof of Lemma 4.2. Because of the term with $\frac{1}{\zeta_{r}}$, the case $0 \leq \zeta_{r} \leq 1$ will be discussed by using (4.9) and (4.10), while the case $\zeta_{r} \geq 1$ will be discussed by using the original form, (4.2) and (4.3). Because the technical details in the proof are similar, we will show here only the part for the case $\zeta_{r} \geq 1$. The proof for the case $0 \leq \zeta_{r} \leq 1$ is left to Appendix A. In the proof, without loss of generality, we assume that $\bar{\zeta}_{1}<\zeta_{1}$.

We start with the proof of (4.17). We denote the integrand of $G$ by $\tilde{g}\left(\zeta_{1}, \zeta_{r}, P, \beta\right)$. It is seen from the definition (4.2) that

$$
\left|\tilde{g}\left(\zeta_{1}, \zeta_{r}, P, \beta\right)-\tilde{g}\left(\bar{\zeta}_{1}, \zeta_{r}, P, \beta\right)\right| \leq \tilde{g}_{1},
$$

where

$$
\tilde{g}_{1}=c_{1}\left(1+\frac{P|\cos \beta|}{\zeta_{r}}\right)\left|\frac{5}{2}-P^{2}-\zeta_{r}^{2}-2 P \zeta_{r} \cos \beta\right| e^{-\frac{\zeta_{r}^{2}}{2}}
$$

On the other hand, by the mean value theorem

$$
\left|\tilde{g}\left(\zeta_{1}, \zeta_{r}, P, \beta\right)-\tilde{g}\left(\bar{\zeta}_{1}, \zeta_{r}, P, \beta\right)\right|=\left|\zeta_{1}-\bar{\zeta}_{1}\right|\left|\frac{\partial \tilde{g}}{\partial \zeta_{1}}\right|_{\zeta_{1}=\zeta_{1}^{\prime}}
$$

where $\zeta_{1}^{\prime}$ is a function of $\zeta_{r}, P$, and $\beta$ taking a value between $\zeta_{1}$ and $\bar{\zeta}_{1}$. Thus, by direct calculation of $\frac{\partial}{\partial \zeta_{1}} \tilde{g}$, we obtain

$$
\left|\tilde{g}\left(\zeta_{1}, \zeta_{r}, P, \beta\right)-\tilde{g}\left(\bar{\zeta}_{1}, \zeta_{r}, P, \beta\right)\right| \leq\left|\zeta_{1}-\bar{\zeta}_{1}\right|\left(\frac{1}{P}\left|\tilde{g}_{2}\right|_{\zeta_{1}=\zeta_{1}^{\prime}}+\left|\tilde{g}_{3}\right|_{\zeta_{1}=\zeta_{1}^{\prime}}\right)
$$

where

$$
\begin{aligned}
& {\left[\begin{array}{l}
\tilde{g}_{2} \\
\tilde{g}_{3}
\end{array}\right]=c_{1}\left[\begin{array}{c}
1+2 \zeta_{r}^{2} \cos ^{2} \beta \\
\zeta_{1}+2 P+4 \zeta_{r} \cos \beta
\end{array}\right]\left(1+\frac{P}{\zeta_{r}} \cos \beta\right) } \\
& \cdot\left(\frac{5}{2}-P^{2}-\zeta_{r}^{2}-2 P \zeta_{r} \cos \beta\right) e^{-\frac{\zeta_{r}^{2}}{2}-\frac{\zeta_{1}^{2}}{2}-\frac{P^{2}}{\zeta_{1}^{2}+P^{2}}\left(P+\zeta_{r} \cos \beta\right)^{2}}
\end{aligned}
$$

We divide the domain of integration into two: $\left(0,\left|\zeta_{1}-\bar{\zeta}_{1}\right|^{\frac{1}{2}}\right) \times I$ and $\left(\mid \zeta_{1}-\right.$ $\left.\left.\bar{\zeta}_{1}\right|^{\frac{1}{2}}, \infty\right) \times I$, where $I=(0,2 \pi)$. Note that both $\zeta_{1}$ and $\bar{\zeta}_{1}$ are less than $\frac{1}{100}$. 
Using (4.19) in the first domain and (4.22) in the second, we obtain

$$
\begin{aligned}
\mid G\left(\zeta_{1}, \zeta_{r}\right) & -G\left(\bar{\zeta}_{1}, \zeta_{r}\right) \mid \\
\leq & \left|\zeta_{1}-\bar{\zeta}_{1}\right|^{\frac{1}{2}}\left(\left\|\tilde{g}_{1}\right\|_{L^{\infty}\left(0, \frac{1}{10}\right) \times I}+\left\|\left.\tilde{g}_{2}\right|_{\zeta_{1}=\zeta_{1}^{\prime}}\right\|_{L^{1}}\right) \\
& +\left|\zeta_{1}-\bar{\zeta}_{1}\right|\left(\left\|\left.\tilde{g}_{3}\right|_{\zeta_{1}=\zeta_{1}^{\prime}}\right\|_{L^{1}}\right) .
\end{aligned}
$$

Below, we list the estimates for the norms in (4.24) for arbitrary $0<\zeta_{1}^{\prime}<\frac{1}{100}$ and $1 \leq \zeta_{r}$ :

$$
\begin{aligned}
& \left|\tilde{g}_{1}\right| \leq c_{1} \frac{11}{10}\left(\zeta_{r}^{2}+\frac{\zeta_{r}}{5}+\frac{251}{100}\right) e^{-\frac{1}{2} \zeta_{r}^{2}}, \quad \text { for } 0 \leq P \leq \frac{1}{10} \\
& \left|\tilde{g}_{2}\right| \leq\left\{\begin{array}{lc}
c_{1}\left(\frac{5}{2}+P^{2}+\zeta_{r}^{2}+2 P \zeta_{r}|\cos \beta|\right)\left(1+2 \zeta_{r}^{2} \cos ^{2} \beta\right)(1+P|\cos \beta|) e^{-\frac{1}{2} \zeta_{r}^{2}}, \\
c_{1} e^{-\frac{1}{2} \zeta_{r}^{2}-\frac{10000}{10001}\left(P-\zeta_{r}\right)^{2}}\left(1+2 \zeta_{r}^{2} \cos ^{2} \beta\right)(1+P|\cos \beta|) & \text { for } 0 \leq P \leq \zeta_{r}, \\
\left(\frac{5}{2}+P^{2}+\zeta_{r}^{2}+2 P \zeta_{r}|\cos \beta|\right), & \text { for } P \geq \zeta_{r},
\end{array}\right. \\
& \left|\tilde{g}_{3}\right| \leq \begin{cases}c_{1}\left(\frac{5}{2}+P^{2}+\zeta_{r}^{2}+2 P \zeta_{r}|\cos \beta|\right)\left(\frac{1}{100}+2 P+4 \zeta_{r}|\cos \beta|\right) & \text { for } 0 \leq P \leq \zeta_{r}, \\
(1+P|\cos \beta|) e^{-\frac{1}{2} \zeta_{r}^{2}}, & \\
c_{1} e^{-\frac{1}{2} \zeta_{r}^{2}-\frac{10000}{10001}\left(P-\zeta_{r}\right)^{2}}\left(\frac{1}{100}+2 P+4 \zeta_{r}|\cos \beta|\right) & \\
\cdot(1+P|\cos \beta|)\left(\frac{5}{2}+P^{2}+\zeta_{r}^{2}+2 \zeta_{r} P|\cos \beta|\right), & \text { for } P \geq \zeta_{r},\end{cases}
\end{aligned}
$$

which are obtained by observing (4.20) and (4.23). Note that the functions on the right hand side above are polynomials of $\zeta_{r}, P$, and $|\cos \beta|$ multiplied by $e^{-\frac{1}{2}\left|\zeta_{r}\right|^{2}}$ or by $e^{-\frac{1}{2}\left|\zeta_{r}\right|^{2}-\frac{10000}{10001}\left(P-\zeta_{r}\right)^{2}}$. Thus, we have

$$
\begin{gathered}
\left|\tilde{g}_{1}\right| \leq C, \quad \text { for } 0 \leq P \leq \frac{1}{10}, \\
{\left[\begin{array}{l}
\tilde{g}_{2} \\
\tilde{g}_{3}
\end{array}\right] \leq\left\{\begin{array}{lc}
C e^{-\frac{1}{4} \zeta_{r}^{2}}, & \text { for } 0 \leq P \leq \zeta_{r}, \\
C e^{-\frac{1}{2}\left|P-\zeta_{r}\right|^{2}}, & \text { for } P \geq \zeta_{r} .
\end{array}\right.}
\end{gathered}
$$


Using the above inequalities, we can conclude $\left\|\tilde{g}_{1}\right\|_{L^{\infty}\left(0, \frac{1}{10}\right) \times I},\left\|\left.\tilde{g}_{2}\right|_{\zeta_{1}=\zeta_{1}^{\prime}}\right\|_{L^{1}}$, and $\left\|\left.\tilde{g}_{3}\right|_{\zeta_{1}=\zeta_{1}^{\prime}}\right\|_{L^{1}}$ are bounded uniformly for $0 \leq \zeta_{1}^{\prime} \leq \frac{1}{100}$ and $\zeta_{r} \geq 1$. Thus, (4.17) is established for $\zeta_{r} \geq 1$.

Next, we turn to the proof of (4.18). We denote the integrand of $H$ by $\tilde{h}\left(\zeta_{1}, \zeta_{r}, P, \beta\right)$. Without loss of generality, we may assume that $\bar{\zeta}_{1}<\zeta_{1}$. Applying the mean value theorem, we have

$$
\left|H\left(\zeta_{1}, \zeta_{r}\right)-H\left(\bar{\zeta}_{1}, \zeta_{r}\right)\right| \leq\left|\zeta_{1}-\bar{\zeta}_{1}\right| \int_{0}^{2 \pi} \int_{0}^{\infty}\left|\frac{\partial}{\partial \zeta_{1}} \tilde{h}\left(\zeta_{1}^{\prime}, \zeta_{r}, P, \beta\right)\right| d P d \beta
$$

where $\bar{\zeta}_{1}<\zeta_{1}^{\prime}<\zeta_{1}$. Calculating $\frac{\partial}{\partial \zeta_{1}} \tilde{h}$ directly and taking account of $\zeta_{r} \geq 1$ and $0 \leq \zeta_{1} \leq \frac{1}{100}$, we have

$$
\left|\frac{\partial}{\partial \zeta_{1}} \tilde{h}\left(\zeta_{1}^{\prime}, \zeta_{r}, P, \beta\right)\right| \leq \begin{cases}C e^{-\frac{\zeta_{r}^{2}}{4}}, & 0 \leq P \leq \zeta_{r}, \\ C e^{-\frac{\left(P-\zeta_{r}\right)^{2}}{2}}, & P \geq \zeta_{r} .\end{cases}
$$

From the above estimate, we see $\left\|\frac{\partial}{\partial \zeta_{1}} \tilde{h}\right\|_{L^{1}}$ is uniformly bounded for $0 \leq \zeta_{1}^{\prime} \leq \frac{1}{100}$ and $\zeta_{r} \geq 1$. Therefore, we obtain (4.18) for $\zeta_{r} \geq 1$.

\section{Contribution from minor terms}

We have so far studied the contribution from $W$, the first term of (2.1) for $n=1$. Now, we are going to establish the estimates for all the four terms on the righthand side of (2.1) for $n \geq 1$ and conclude Lemma 2.5. We will use the following lemma that has been established in [3].

Lemma 5.1. For $0 \leq a<\frac{1}{2}$ and $\kappa$ large enough (depending on $a$ ), $n \geq 0$, there exist positive constants $C$, and $C_{a}$ and $C_{a}^{\prime}$ depending on a, such that

$$
\left|\partial_{s} K\left(\phi_{n}\right)\right| \leq C\left(C_{a} \frac{\ln \kappa}{\kappa}\right)^{n}\left(\ln \kappa+|\ln | s+\frac{1}{2}||+|\ln | s-\frac{1}{2}||\right),
$$




$$
\begin{gathered}
\left|\phi_{n}\right| \leq C_{a}^{\prime}\left(\frac{C_{a} \ln \kappa}{\kappa}\right)^{n}\left(\kappa^{-1}+\left|\zeta_{1}\right|\right)^{-1} e^{-a|\zeta|^{2}}, \\
\left|K\left(\phi_{n}\right)\right| \leq C_{a}^{\prime}\left(\frac{C_{a} \ln \kappa}{\kappa}\right)^{n+1} \kappa e^{-a|\zeta|^{2}} .
\end{gathered}
$$

For the first term of (2.1), by using Lemma 2.1 and (5.1), we obtain

\section{Lemma 5.2.}

$$
\begin{aligned}
& \mid \frac{1}{\kappa\left|\zeta_{1}\right|^{2}} \int_{-\frac{1}{2}}^{\frac{1}{2}} e^{-\frac{\nu}{\kappa\left|\zeta_{1}\right|}\left(\frac{1}{2}-s\right)}\left(\frac{1}{2}\right.-s) \partial_{s} K\left(\phi_{n-1}\right) d s \mid \\
& \leq C \frac{\kappa}{\nu^{2}}\left(\left|\ln \frac{\kappa\left|\zeta_{1}\right|}{\nu}\right|+\ln \kappa\right)\left(\frac{C_{a} \ln \kappa}{\kappa}\right)^{n-1} .
\end{aligned}
$$

For the second term of (2.1), by using (5.3), we have

$$
\begin{aligned}
\left|\frac{1}{\kappa\left|\zeta_{1}\right|^{2}} e^{-\frac{\nu}{\kappa\left|\zeta_{1}\right|}} K\left(\phi_{n-1}\right)\left(-\frac{1}{2}, \zeta\right)\right| & \leq \frac{C_{a}^{\prime}}{\left|\zeta_{1}\right|^{2}} e^{-\frac{\nu}{\kappa \mid \zeta_{1}}}\left(\frac{C_{a} \ln \kappa}{\kappa}\right)^{n} e^{-a|\zeta|^{2}} \\
& \leq C_{a}^{\prime} C\left(\frac{C_{a} \ln \kappa}{\kappa}\right)^{n} e^{-a|\zeta|^{2}},
\end{aligned}
$$

where we use the following estimate in the last inequality:

$$
\left|\frac{1}{\zeta_{1}^{2}} e^{-\frac{\nu}{\kappa \zeta_{1}}}\right| \leq \frac{1}{\zeta_{1}^{2}} e^{-O(1) \zeta_{1}^{-\frac{1}{2}}}<C, \quad \text { for } 0<\zeta_{1}<\frac{1}{\kappa^{2}} .
$$

For the third term of (2.1), we use (5.3) and the bound

$$
\left|\frac{\nu^{\prime}(|\zeta|)}{|\zeta|}\right|<C
$$

to obtain

$$
\begin{aligned}
& \left|\int_{-\frac{1}{2}}^{\frac{1}{2}} \frac{1}{\kappa\left|\zeta_{1}\right|} e^{-\frac{\nu}{\kappa\left|\zeta_{1}\right|}\left|\frac{1}{2}-s\right|} \frac{\nu^{\prime}\left|\frac{1}{2}-s\right|}{\kappa|\zeta|} K\left(\phi_{n-1}\right) d s\right| \\
\leq & C C_{a}^{\prime} \int_{-\frac{1}{2}}^{\frac{1}{2}} \frac{\left|\frac{1}{2}-s\right|}{\kappa\left|\zeta_{1}\right|} e^{-\frac{\nu}{\kappa\left|\zeta_{1}\right|}\left|\frac{1}{2}-s\right|} d s\left(\frac{C_{a} \ln \kappa}{\kappa}\right)^{n} e^{-a|\zeta|^{2}} \leq \frac{C C_{a}^{\prime}}{\nu}\left(\frac{C_{a} \ln \kappa}{\kappa}\right)^{n} e^{-a|\zeta|^{2}} .
\end{aligned}
$$

Here, the boundedness of $\nu^{\prime}(\eta) / \eta$ for $\eta \geq 0$ is readily confirmed of its form that is obtained by direct calculation:

$$
\frac{\nu^{\prime}(\eta)}{\eta}=2^{-\frac{1}{2}}\left(\frac{1}{\eta} \int_{0}^{\eta} e^{-z^{2}} d z-\frac{1}{\eta^{3}} \int_{0}^{\eta} z^{2} e^{-z^{2}} d z\right) .
$$


For the last term of (2.1), we have the following estimate:

Lemma 5.3. For large enough $\kappa$

$$
\left|\int_{-\frac{1}{2}}^{\frac{1}{2}} \frac{1}{\kappa\left|\zeta_{1}\right|} e^{-\frac{\nu}{\kappa\left|\zeta_{1}\right|}\left|\frac{1}{2}-s\right|} \partial_{\zeta_{i}} K\left(\phi_{n-1}\right) d s\right| \leq C_{a}^{\prime}\left(\frac{C_{a} \ln \kappa}{\kappa}\right)^{n-1} \kappa e^{-a|\zeta|^{2}}
$$

where $0<a<\frac{1}{2}$.

Proof. Consider the case $i=1$. Recalling the definition of $k_{1}\left(\zeta, \zeta_{*}\right)$ in $(1.9)$, by direct calculation, we have

$$
\begin{aligned}
\frac{\partial k_{1}}{\partial \zeta_{1}}= & \frac{1}{\sqrt{2} \pi}\left(\frac{\left(|\zeta|^{2}-\left|\zeta_{*}\right|^{2}\right)^{2}}{2\left|\zeta-\zeta_{*}\right|^{4}}-\frac{1}{\left|\zeta-\zeta_{*}\right|^{2}}-\frac{1}{2}\right) \frac{\zeta_{1}-\zeta_{* 1}}{\left|\zeta-\zeta_{*}\right|} e^{-\frac{\left(|\zeta|^{2}-\left|\zeta_{*}\right|^{2}\right)^{2}}{4\left|\zeta-\zeta_{*}\right|^{2}}-\frac{1}{4}\left|\zeta-\zeta_{*}\right|^{2}} \\
& -\frac{1}{\sqrt{2} \pi} \frac{|\zeta|^{2}-\left|\zeta_{*}\right|^{2}}{\left|\zeta-\zeta_{*}\right|^{3}} \zeta_{1} e^{-\frac{\left(|\zeta|^{2}-\left|\zeta_{*}\right|^{2}\right)^{2}}{4\left|\zeta-\zeta_{*}\right|^{2}}-\frac{1}{4}\left|\zeta-\zeta_{*}\right|^{2}}
\end{aligned}
$$

Noting that

$$
\frac{\left|\zeta_{1}-\zeta_{* 1}\right|}{\left|\zeta-\zeta_{*}\right|} \leq 1
$$

and

$$
\frac{\left(|\zeta|^{2}-\left|\zeta_{*}\right|^{2}\right)^{2}}{\left|\zeta-\zeta_{*}\right|^{4}} \leq \frac{\left(|\zeta|+\left|\zeta_{*}\right|\right)^{2}}{\left|\zeta-\zeta_{*}\right|^{2}} \leq \frac{\left(\left|\zeta-\zeta_{*}\right|+2|\zeta|\right)^{2}}{\left|\zeta-\zeta_{*}\right|^{2}} \leq \frac{2\left|\zeta-\zeta_{*}\right|^{2}+8|\zeta|^{2}}{\left|\zeta-\zeta_{*}\right|^{2}}
$$

we obtain

$$
\left|\frac{\partial k_{1}}{\partial \zeta_{1}}\right| \leq C\left(1+\frac{|\zeta|^{2}+1}{\left|\zeta-\zeta_{*}\right|^{2}}\right) e^{-\frac{\left(|\zeta|^{2}-|\zeta|^{2}\right)^{2}}{4\left|\zeta-\zeta_{*}\right|^{2}}-\frac{1}{4}\left|\zeta-\zeta_{*}\right|^{2}}
$$

For $k_{2}\left(\zeta, \zeta_{*}\right)$, by direct calculation, we have

$$
\begin{aligned}
\left|\frac{\partial k_{2}}{\partial \zeta_{1}}\right| & =2^{-\frac{3}{2}} \pi^{-1}\left|\frac{\zeta_{1}-\zeta_{* 1}}{\left|\zeta-\zeta_{*}\right|}-\right| \zeta-\zeta_{*}\left|\zeta_{1}\right| e^{-\frac{1}{2}\left(|\zeta|^{2}+\left|\zeta_{*}^{2}\right|\right)} \\
& \leq C\left(1+|\zeta|^{2}+|\zeta|\left|\zeta_{*}\right|\right) e^{-\frac{1}{2}\left(|\zeta|^{2}+\left|\zeta_{*}^{2}\right|\right)}
\end{aligned}
$$

Recalling now the estimate (5.2), for large enough $\kappa$

$$
\left|\phi_{n-1}\right| \leq C_{a}^{\prime}\left(C_{a} \frac{\ln \kappa}{\kappa}\right)^{n-1}\left(\kappa^{-1}+\left|\zeta_{1}\right|\right)^{-1} e^{-a|\zeta|^{2}} \leq C_{a}^{\prime}\left(C_{a} \frac{\ln \kappa}{\kappa}\right)^{n-1} \kappa e^{-a|\zeta|^{2}}
$$


we have

$$
\left|\partial_{\zeta_{1}} K\left(\phi_{n-1}\right)\right| \leq C_{a}^{\prime}\left(C_{a} \frac{\ln \kappa}{\kappa}\right)^{n-1} \kappa \int\left(\left|\frac{\partial k_{1}}{\partial \zeta_{1}}\right|+\left|\frac{\partial k_{2}}{\partial \zeta_{1}}\right|\right) e^{-a\left|\zeta_{*}\right|^{2}} d \zeta_{*} .
$$

The latter term in (5.12) is readily estimated by using (5.11) as

$$
\int\left|\frac{\partial k_{2}}{\partial \zeta_{1}}\right| e^{-a\left|\zeta_{*}\right|^{2}} d \zeta_{*} \leq \int\left|\frac{\partial k_{2}}{\partial \zeta_{1}}\right| d \zeta_{*} \leq C\left(1+|\zeta|^{2}+|\zeta|\right) e^{-\frac{1}{2}|\zeta|^{2}} \leq C_{a}^{\prime \prime} e^{-a|\zeta|^{2}} .
$$

To handle the former term in (5.12), we choose an orthogonal basis $\left\{e_{1}, e_{2}, e_{3}\right\}$ centered at $\zeta$ with $e_{3}=-\frac{\zeta}{|\zeta|}$ as the north pole and $(\rho, \theta, \varphi)$ its spherical coordinates:

$$
\zeta_{*}=\zeta+\rho \cos \theta e_{3}+\rho \sin \theta \cos \varphi e_{1}+\rho \sin \theta \sin \varphi e_{2} .
$$

Then, using (5.10), we have

$$
\begin{aligned}
& \int\left|\frac{\partial k_{1}}{\partial \zeta_{1}}\right| e^{-a\left|\zeta_{*}\right|^{2}} d \zeta_{*} \\
& \leq C \int\left(\rho^{2}+|\zeta|^{2}+1\right) e^{-a|\zeta|^{2}-\left(a+\frac{1}{2}\right)(\rho-|\zeta| \cos \theta)^{2}+\left(a-\frac{1}{2}\right)|\zeta|^{2} \cos ^{2} \theta} \sin \theta d \rho d \theta d \varphi \\
& \leq C^{\prime} e^{-a|\zeta|^{2}} \int\left[(\rho-|\zeta| \cos \theta)^{2}+|\zeta|^{2}+1\right] \\
& \cdot e^{-\left(a+\frac{1}{2}\right)(\rho-|\zeta| \cos \theta)^{2}-\left(\frac{1}{2}-a\right)|\zeta|^{2} \cos ^{2} \theta} \sin \theta d \rho d \theta \\
& \leq C^{\prime \prime} C_{a} e^{-a|\zeta|^{2}} \int\left(|\zeta|^{2}+1\right) e^{-\left(\frac{1}{2}-a\right)|\zeta|^{2} \cos ^{2} \theta} \sin \theta d \theta .
\end{aligned}
$$

The last integral is bounded by a positive constant for $|\zeta| \leq 1$, while it is bounded by $(1+|\zeta|)\left(\frac{1}{2}-a\right)^{-\frac{1}{2}}$ for $|\zeta|>1$. Therefore we obtain

$$
\left|\partial_{\zeta_{1}} K\left(\phi_{n-1}\right)\right| \leq C_{a}^{\prime}\left(C_{a} \frac{\ln \kappa}{\kappa}\right)^{n-1} \kappa(1+|\zeta|) e^{-a|\zeta|^{2}} .
$$

The same estimate holds for the cases $i=2$ and 3 . Thus we conclude

$$
\begin{aligned}
& \left|\int_{-\frac{1}{2}}^{\frac{1}{2}} \frac{1}{\kappa\left|\zeta_{1}\right|} e^{-\frac{\nu}{\kappa\left|\zeta_{1}\right|}\left|\frac{1}{2}-s\right|} \partial_{\zeta_{i}} K\left(\phi_{n-1}\right) d s\right| \\
& \quad \leq C_{a}^{\prime}\left(C_{a} \frac{\ln \kappa}{\kappa}\right)^{n-1} \kappa(1+|\zeta|) e^{-a|\zeta|^{2}} \int_{-\frac{1}{2}}^{\frac{1}{2}} \frac{1}{\kappa\left|\zeta_{1}\right|} e^{-\frac{\nu}{\kappa\left|\zeta_{1}\right|}\left|\frac{1}{2}-s\right|} d s \\
& \quad \leq C_{a}^{\prime}\left(C_{a} \frac{\ln \kappa}{\kappa}\right)^{n-1} e^{-a|\zeta|^{2}} \kappa .
\end{aligned}
$$


Here we have used the fact from (1.10) that

$$
\int_{-\frac{1}{2}}^{\frac{1}{2}} \frac{1}{\kappa\left|\zeta_{1}\right|} e^{-\frac{\nu}{\kappa \mid \zeta_{1}}\left|\frac{1}{2}-s\right|} d s=\frac{1}{\nu}\left(1-e^{\left.-\frac{\nu}{\kappa \mid \zeta_{1}}\right)}\right)<\frac{1}{\nu_{0}(1+|\zeta|)}
$$

With the estimates above, we can present the proof of Lemma 2.5, from which Theorem 1.1 is finally established.

Proof of Lemma 2.5. Because of (1.10), we have

$$
e^{-\frac{|\zeta|^{2}}{4}}<C\left(\frac{1}{\nu}\right), \quad e^{-\frac{|\zeta|^{2}}{4}}<C\left(\frac{1}{\nu^{2}}\right)
$$

With these inequalities, applying (5.4), (5.5), (5.8), and (5.9) with $a=\frac{1}{4}$ to (2.1), we conclude $(2.6)$ and (2.7). It remains to prove (2.8). We calculate $\frac{\partial}{\partial \zeta_{1}} \phi_{0}$ at $x_{1}=\frac{1}{2}$ directly to obtain

$$
\begin{aligned}
\frac{\partial \phi_{0}}{\partial \zeta_{1}}\left(\frac{1}{2}, \zeta\right)= & -\frac{\kappa}{\nu^{2}} \frac{\nu^{\prime}}{|\zeta|}\left(1-e^{-\frac{\nu}{\kappa \zeta_{1}}}\right) \zeta_{1} \zeta_{2}\left(\frac{5}{2}-|\zeta|^{2}\right) \pi^{-\frac{3}{4}} e^{-\frac{|\zeta|^{2}}{2}} \\
& +\frac{\kappa}{\nu}\left(1-e^{-\frac{\nu}{\kappa \zeta_{1}}}\right) \zeta_{1} \zeta_{2}\left(|\zeta|^{2}-\frac{9}{2}\right) \pi^{-\frac{3}{4}} e^{-\frac{|\zeta|^{2}}{2}} \\
& +\frac{1}{\nu} \frac{\nu^{\prime}}{|\zeta|} e^{-\frac{\nu}{\kappa \zeta_{1}}} \zeta_{2}\left(\frac{5}{2}-|\zeta|^{2}\right) \pi^{-\frac{3}{4}} e^{-\frac{|\zeta|^{2}}{2}} \\
& -\frac{1}{\zeta_{1}^{2}} e^{-\frac{\nu}{\kappa \zeta_{1}}} \zeta_{2}\left(\frac{5}{2}-|\zeta|^{2}\right) \pi^{-\frac{3}{4}} e^{-\frac{|\zeta|^{2}}{2}}
\end{aligned}
$$

From (5.7) and (5.14), the first three terms on the right-hand side are bounded by $C \frac{\kappa}{\nu^{2}}$. Because of (5.6), the last term is also bounded by $C \frac{\kappa}{\nu^{2}}$. Therefore, we have (2.8).

Finally we confirm the claim in the Remark 1.1, that is,

Lemma 5.4. If $0<\zeta_{1}<\frac{1}{\kappa^{2}}<\frac{1}{100}$ and $|\zeta|=0.5$ and $\kappa$ large enough, then

$$
\left|\frac{\zeta_{1}}{\sqrt{\zeta_{2}^{2}+\zeta_{3}^{2}}} \cos \theta \frac{\partial}{\partial \zeta_{2}} \phi\left(\frac{1}{2}, \zeta\right)+\frac{\zeta_{1}}{\sqrt{\zeta_{2}^{2}+\zeta_{3}^{2}}} \sin \theta \frac{\partial}{\partial \zeta_{3}} \phi\left(\frac{1}{2}, \zeta\right)\right| \leq C \frac{\kappa}{\nu^{2}}
$$


Proof. By direct computation,

$$
\begin{aligned}
& \left|\frac{\zeta_{1}}{\sqrt{\zeta_{2}^{2}+\zeta_{3}^{2}}} \cos \theta \frac{\partial}{\partial \zeta_{2}} \phi_{0}\left(\frac{1}{2}, \zeta\right)\right|=\mid \pi^{-\frac{3}{4}} \frac{\cos \theta}{\sqrt{\zeta_{2}^{2}+\zeta_{3}^{2}}} e^{-\frac{|\zeta|^{2}}{2}}\left[\frac{1}{\nu} \frac{\nu^{\prime}}{|\zeta|} \zeta_{2}^{2}\left(\frac{5}{2}-|\zeta|^{2}\right) e^{-\frac{\nu}{\kappa \zeta_{1}}}\right. \\
& +\frac{\kappa}{\nu}\left(1-e^{-\frac{\nu}{\kappa \zeta_{1}}}\right) \zeta_{1}\left(\left(\frac{5}{2}-|\zeta|^{2}\right)+\zeta_{2}^{2}\left(|\zeta|^{2}-\frac{9}{2}\right)\right) \\
& \left.-\frac{\kappa}{\nu^{2}} \frac{\nu^{\prime}}{|\zeta|}\left(1-e^{-\frac{\nu}{\kappa \zeta_{1}}}\right) \zeta_{1} \zeta_{2}^{2}\left(\frac{5}{2}-|\zeta|^{2}\right)\right] \mid \\
& \leq C \frac{\kappa}{\nu^{2}}
\end{aligned}
$$

where we have used (5.7) and (5.14) and the fact that when $|\zeta|=0.5$ and $0<\zeta_{1}<\frac{1}{100}$

$$
\zeta_{2}^{2}+\zeta_{3}^{2}>0.2499
$$

Similarly,

$$
\begin{aligned}
& \left|\frac{\zeta_{1}}{\sqrt{\zeta_{2}^{2}+\zeta_{3}^{2}}} \sin \theta \frac{\partial}{\partial \zeta_{3}} \phi_{0}\left(\frac{1}{2}, \zeta\right)\right|=\mid \pi^{-\frac{3}{4}} \frac{\sin \theta}{\sqrt{\zeta_{2}^{2}+\zeta_{3}^{2}}} e^{\frac{|\zeta|^{2}}{2}}\left[\frac{1}{\nu} \frac{\nu^{\prime}}{|\zeta|} \zeta_{2} \zeta_{3}\left(\frac{5}{2}-|\zeta|^{2}\right) e^{-\frac{\nu}{\kappa \zeta_{1}}}\right. \\
& \left.+\frac{\kappa}{\nu}\left(1-e^{-\frac{\nu}{\kappa \zeta_{1}}}\right) \zeta_{1} \zeta_{2} \zeta_{3}\left(|\zeta|^{2}-\frac{9}{2}\right)-\frac{\kappa}{\nu^{2}} \frac{\nu^{\prime}}{|\zeta|}\left(1-e^{-\frac{\nu}{\kappa \zeta_{1}}}\right) \zeta_{1} \zeta_{2} \zeta_{3}\left(\frac{5}{2}-|\zeta|^{2}\right)\right] \mid \\
& \leq C \frac{\kappa}{\nu^{2}} .
\end{aligned}
$$

For higher order terms, we calculate directly as

$$
\begin{aligned}
& \left|\frac{\zeta_{1}}{\sqrt{\zeta_{2}^{2}+\zeta_{3}^{2}}} \cos \theta \frac{\partial}{\partial \zeta_{2}} \phi_{n}\left(\frac{1}{2}, \zeta\right)\right| \\
= & \mid \frac{\cos \theta}{\sqrt{\zeta_{2}^{2}+\zeta_{3}^{2}}}\left[-\int_{-\frac{1}{2}}^{\frac{1}{2}} \frac{1}{\kappa\left|\zeta_{1}\right|} e^{-\frac{\nu}{\kappa \zeta_{1}}\left(\frac{1}{2}-s\right)} \frac{\nu^{\prime}\left|\frac{1}{2}-s\right|}{\kappa|\zeta|} \zeta_{2} K\left(\phi_{n-1}\right) d s\right. \\
& \left.+\int_{-\frac{1}{2}}^{\frac{1}{2}} \frac{1}{\kappa} e^{-\frac{\nu}{\kappa \zeta_{1}}\left(\frac{1}{2}-s\right)} \partial_{\zeta_{2}} K\left(\phi_{n-1}\right) d s\right] \mid \\
\leq & \frac{C C_{a}^{\prime}}{\nu}\left(\frac{C_{a} \ln \kappa}{\kappa}\right)^{n}\left|\zeta_{2}\right| e^{-a|\zeta|^{2}}+C_{a}^{\prime}\left(\frac{C_{a} \ln \kappa}{\kappa}\right)^{n-1}\left|\zeta_{1}\right| e^{-a|\zeta|^{2}} \kappa,
\end{aligned}
$$

where we have used (5.8) and (5.9) together with (5.17) to obtain the last inequality. Therefore, we have

$$
\left|\frac{\zeta_{1}}{\sqrt{\zeta_{2}^{2}+\zeta_{3}^{2}}} \cos \theta \frac{\partial}{\partial \zeta_{2}} \phi_{n}\left(\frac{1}{2}, \zeta\right)\right| \leq C \frac{\kappa}{\nu^{2}}\left(\frac{\ln \kappa}{\kappa}\right)^{n-1}
$$


and, similarly,

$$
\left|\frac{\zeta_{1}}{\sqrt{\zeta_{2}^{2}+\zeta_{3}^{2}}} \sin \theta \frac{\partial}{\partial \zeta_{3}} \phi_{n}\left(\frac{1}{2}, \zeta\right)\right| \leq C \frac{\kappa}{\nu^{2}}\left(\frac{\ln \kappa}{\kappa}\right)^{n-1}
$$

Combining estimates above, if $\kappa$ is large enough, we can conclude the lemma.

\section{A. Proof of (4.17) and (4.18) for $0 \leq \zeta_{r} \leq 1$}

We discuss the continuity estimates for $0 \leq \zeta_{r} \leq 1$ in Lemma 4.2 .

We start with the proof of (4.17), treating $G_{1}$ and $G_{2}$ parts in $G$ separately. First consider $G_{1}\left(\zeta_{1}, \zeta_{r}\right)-G_{1}\left(\bar{\zeta}_{1}, \zeta_{r}\right)$ and denote its integrand by $\Delta_{1}$. On one hand, we have from (4.9)

$$
\left|\Delta_{1}\right| \leq 4 \pi c_{1}\left(\left|\frac{5}{2}-2 P^{2}\right|+P^{2}\left|\frac{5}{2}-P^{2}\right|\right) e^{-\frac{P^{4}}{1+P^{2}}}=: g_{1} \text {. }
$$

On the other hand, by the mean value theorem, we have for some $\bar{\zeta}_{1}<\zeta_{1}^{\prime}<\zeta_{1}$

$$
\begin{aligned}
\left|\Delta_{1}\right| \leq & \left|\zeta_{1}-\bar{\zeta}_{1}\right| 2 \pi c_{1} e^{-\frac{\zeta_{1}^{\prime 2}}{2}-\frac{P^{4}}{\zeta_{1}^{\prime 2}+P^{2}}} \mid-\frac{P \zeta_{1}^{\prime}\left(\frac{5}{2}-2 P^{2}\right)}{\left(\zeta_{1}^{\prime 2}+P^{2}\right)^{\frac{3}{2}}}+\frac{3 P^{5} \zeta_{1}^{\prime}\left(\frac{5}{2}-P^{2}\right)}{\left(\zeta_{1}^{\prime 2}+P^{2}\right)^{\frac{5}{2}}} \\
& +\left(\frac{2 P^{4}}{\left(\zeta_{1}^{\prime 2}+P^{2}\right)^{2}}-1\right) \zeta_{1}^{\prime}\left(\frac{P\left(\frac{5}{2}-2 P^{2}\right)}{\left(\zeta_{1}^{\prime 2}+P^{2}\right)^{\frac{1}{2}}}-\frac{P^{5}\left(\frac{5}{2}-P^{2}\right)}{\left(\zeta_{1}^{\prime 2}+P^{2}\right)^{\frac{3}{2}}}\right) \mid \\
\leq & \left|\zeta_{1}-\bar{\zeta}_{1}\right| 2 \pi c_{1} e^{-\frac{P^{4}}{1+P^{2}}}\left(\frac{1}{P}\left|\frac{5}{2}-2 P^{2}\right|+\frac{3}{100}\left|\frac{5}{2}-2 P^{2}\right|\right. \\
& \left.+\frac{3}{100}\left(P^{2}+1\right)\left|\frac{5}{2}-P^{2}\right|\right)=:\left|\zeta_{1}-\bar{\zeta}_{1}\right|\left(\frac{1}{P} g_{2}+g_{3}\right),
\end{aligned}
$$

where

$$
\begin{aligned}
& g_{2}=2 \pi c_{1} e^{-\frac{P^{4}}{1+P^{2}}}\left|\frac{5}{2}-2 P^{2}\right|, \\
& g_{3}=\frac{3}{50} \pi c_{1} e^{-\frac{P^{4}}{1+P^{2}}}\left(\left|\frac{5}{2}-2 P^{2}\right|+\left(P^{2}+1\right)\left|\frac{5}{2}-P^{2}\right|\right) .
\end{aligned}
$$

We divide the domain of integration into two: $\left(0,\left|\zeta_{1}-\bar{\zeta}_{1}\right|^{\frac{1}{2}}\right)$ and $\left(\left|\zeta_{1}-\bar{\zeta}_{1}\right|^{\frac{1}{2}}, \infty\right)$.

We use the former estimate (A.1) in the first domain and the latter estimate 
(A.2) in the second to obtain

$$
\begin{aligned}
& \left|G_{1}\left(\zeta_{1}, \zeta_{r}\right)-G_{1}\left(\bar{\zeta}_{1}, \zeta_{r}\right)\right| \\
& \quad \leq\left|\zeta_{1}-\bar{\zeta}_{1}\right|^{\frac{1}{2}}\left(\left\|g_{1}\right\|_{L_{P}^{\infty}\left(0, \frac{1}{10}\right)}+\left\|g_{2}\right\|_{L_{P}^{1}}\right)+\left|\zeta_{1}-\bar{\zeta}_{1}\right| \cdot\left\|g_{3}\right\|_{L_{P}^{1}} .
\end{aligned}
$$

From the expressions in (A.1), (A.3), and (A.4), we see that $\left\|g_{1}\right\|_{L_{P}^{\infty}\left(0, \frac{1}{10}\right)}$, $\left\|g_{2}\right\|_{L_{P}^{1}}$, and $\left\|g_{3}\right\|_{L_{P}^{1}}$ are bounded.

Next consider $G_{2}\left(\zeta_{1}, \zeta_{r}\right)-G_{2}\left(\bar{\zeta}_{1}, \zeta_{r}\right)$ and rewrite it as

$$
\begin{aligned}
& G_{2}\left(\zeta_{1}, \zeta_{r}\right)-G_{2}\left(\bar{\zeta}_{1}, \zeta_{r}\right)=\int_{0}^{2 \pi} \int_{0}^{\infty} \Delta_{21} R_{g}\left(\zeta_{1}, \zeta_{r}, P, \beta\right)\left(1+\frac{P}{\zeta_{r}} \cos \beta\right) d P d \beta \\
&+\int_{0}^{2 \pi} \int_{0}^{\infty} \frac{c_{1} P}{\sqrt{\bar{\zeta}_{1}^{2}+P^{2}}} e^{-\frac{\bar{\zeta}_{1}^{2}}{2}-\frac{P^{4}}{\bar{\zeta}_{1}^{2}+P^{2}}} \Delta_{22}\left(1+\frac{P}{\zeta_{r}} \cos \beta\right) d P d \beta
\end{aligned}
$$

where

$$
\begin{aligned}
& \Delta_{21}=\frac{c_{1} P}{\sqrt{\zeta_{1}^{2}+P^{2}}} e^{-\frac{\zeta_{1}^{2}}{2}-\frac{P^{4}}{\zeta_{1}^{2}+P^{2}}}-\frac{c_{1} P}{\sqrt{\bar{\zeta}_{1}^{2}+P^{2}}} e^{-\frac{\bar{\zeta}_{1}^{2}}{2}-\frac{P^{4}}{\bar{\zeta}_{1}^{2}+P^{2}}} \\
& \Delta_{22}=R_{g}\left(\zeta_{1}, \zeta_{r}, P, \beta\right)-R_{g}\left(\bar{\zeta}_{1}, \zeta_{r}, P, \beta\right) .
\end{aligned}
$$

On one hand, we obviously have

$$
\left|\Delta_{21}\right| \leq c_{1} e^{-\frac{P^{4}}{\zeta_{1}^{2}+P^{2}}}
$$

On the other hand, again by the mean value theorem, for some $\bar{\zeta}_{1}<\zeta_{1}^{\prime}<\zeta_{1}$ we have

$$
\begin{aligned}
\left|\Delta_{21}\right| & =\left|\zeta_{1}-\bar{\zeta}_{1}\right| e^{-\frac{\zeta_{1}^{\prime 2}}{2}-\frac{P^{4}}{\zeta_{1}^{\prime 2}+P^{2}}}\left|\frac{c_{1} P \zeta_{1}^{\prime}}{\left(\zeta_{1}^{\prime 2}+P^{2}\right)^{\frac{1}{2}}} \frac{P^{4}-2 P^{2} \zeta_{1}^{\prime 2}-\zeta_{1}^{\prime 4}}{P^{4}+2 P^{2} \zeta_{1}^{\prime 2}+\zeta_{1}^{\prime 4}}-\frac{c_{1} P \zeta_{1}^{\prime}}{\left(\zeta_{1}^{\prime 2}+P^{2}\right)^{\frac{3}{2}}}\right| \\
& \leq c_{1} e^{-\frac{P^{4}}{\zeta_{1}^{2}+P^{2}}}\left|\zeta_{1}-\bar{\zeta}_{1}\right|\left(\frac{1}{P}+\frac{1}{100}\right) .
\end{aligned}
$$

We divide the domain of integration into two: $\left(0,\left|\zeta_{1}-\bar{\zeta}_{1}\right|^{\frac{1}{2}}\right) \times I$ and $\left(\mid \zeta_{1}-\right.$ $\left.\left.\bar{\zeta}_{1}\right|^{\frac{1}{2}}, \infty\right) \times I$, where $I=(0,2 \pi)$. We apply the former estimate (A.6) in $\left(0, \mid \zeta_{1}-\right.$ 
$\left.\left.\bar{\zeta}_{1}\right|^{\frac{1}{2}}\right) \times I$ and the latter estimate (A.7) in the other domain. Then we obtain

$$
\begin{aligned}
\mid \int_{0}^{2 \pi} \int_{0}^{\infty} \Delta_{21} & \left(1+\frac{P}{\zeta_{r}} \cos \beta\right) R_{g}\left(\zeta_{1}, \zeta_{r}, P, \beta\right) d P d \beta \mid \\
\leq & \left|\zeta_{1}-\bar{\zeta}_{1}\right|^{\frac{1}{2}}\left(\left\|g_{4}\right\|_{L^{\infty}\left(0, \frac{1}{10}\right) \times I}+\left\|g_{4}\right\|_{L^{1}}\right) \\
& +\left|\zeta_{1}-\bar{\zeta}_{1}\right|\left(\frac{1}{100}\left\|g_{4}\right\|_{L^{1}}\right)
\end{aligned}
$$

where

$$
g_{4}\left(\zeta_{1}, \zeta_{r}, P, \beta\right)=c_{1} e^{-\frac{P^{4}}{\zeta_{1}^{2}+P^{2}}}\left(1+\frac{P}{\zeta_{r}} \cos \beta\right) R_{g}\left(\zeta_{1}, \zeta_{r}, P, \beta\right) .
$$

Remind that $g_{4}$ contains $g$ through $R_{g}$ in its definition, see (4.11) and (4.7).

Applying the mean value theorem to one of the terms in $g$ :

$$
\begin{aligned}
\left(\frac{5}{2}-P^{2}\right) e^{-\frac{1}{2} \zeta_{r}^{2}-\frac{P^{2}}{\zeta_{1}^{2}+P^{2}}\left(2 \zeta_{r} P \cos \beta+\zeta_{r}^{2} \cos ^{2} \beta\right)}=\frac{5}{2}-P^{2}-\zeta_{r}\left(\frac{5}{2}-P^{2}\right)\left(\zeta_{r}^{\prime}\right. \\
\left.+\frac{2 P^{2}}{\zeta_{1}^{2}+P^{2}}\left(P \cos \beta+\zeta_{r}^{\prime} \cos ^{2} \beta\right)\right) e^{-\frac{1}{2} \zeta_{r}^{\prime 2}-\frac{P^{2}}{\zeta_{1}^{2}+P^{2}}\left(2 \zeta_{r}^{\prime} P \cos \beta+\zeta_{r}^{\prime 2} \cos ^{2} \beta\right)},
\end{aligned}
$$

for some $0<\zeta_{r}^{\prime}<\zeta_{r}$ and keeping the other term in $g$ as it is, we obtain from

$$
\begin{aligned}
g_{4} & =c_{1}\left(\zeta_{r}+P \cos \beta\right)\left(2 P \cos \beta\left[1+\frac{P^{2}}{\zeta_{1}^{2}+P^{2}}\left(\frac{5}{2}-P^{2}\right)\right] e^{-\frac{P^{4}}{\zeta_{1}^{2}+P^{2}}}\right. \\
& -\left(\zeta_{r}+2 P \cos \beta\right) e^{-\frac{\zeta_{r}^{2}}{2}-\frac{P^{2}}{\zeta_{1}^{2}+P^{2}}\left(\zeta_{r} \cos \beta+P\right)^{2}} \\
& \left.-\left(\frac{5}{2}-P^{2}\right)\left[\zeta_{r}^{\prime}+\frac{2 P^{2}}{\zeta_{1}^{2}+P^{2}}\left(P \cos \beta+\zeta_{r}^{\prime} \cos ^{2} \beta\right)\right] e^{-\frac{\zeta_{r}^{\prime 2}}{2}-\frac{P^{2}}{\zeta_{1}^{2}+P^{2}}\left(\zeta_{r}^{\prime} \cos \beta+P\right)^{2}}\right) .
\end{aligned}
$$

Therefore, because $0 \leq \zeta_{r} \leq 1$ and $0 \leq \zeta_{1} \leq \frac{1}{100}$, we obtain the following estimates for $g_{4}$ :

$$
\left|g_{4}\right| \leq \begin{cases}c_{1}(1+P|\cos \beta|)(1+4 P|\cos \beta| & \text { for } 0<P<1, \\ & \left.+\left(\frac{5}{2}-P^{2}\right)\left(1+4 P|\cos \beta|+2 \cos ^{2} \beta\right)\right), \\ 2 c_{1} e^{-\frac{10000}{10001} P^{2}} P|\cos \beta|(1+P|\cos \beta|)\left(1+\frac{5}{2}+P^{2}\right) & \\ +c_{1} e^{-\frac{10000}{10001}(P-1)^{2}}(1+P|\cos \beta|) & \\ \cdot\left(1+2 P|\cos \beta|+\left(\frac{5}{2}+P^{2}\right)\left(1+2 P|\cos \beta|+2|\cos \beta|^{2}\right)\right), \text { for } 1 \leq P,\end{cases}
$$


and see that $\left\|g_{4}\right\|_{L^{\infty}\left(0, \frac{1}{10}\right) \times I}$ and $\left\|g_{4}\right\|_{L^{1}}$ are bounded.

As to $\Delta_{22}$, again by the mean value theorem, we have for some $\bar{\zeta}_{1}<\zeta_{1}^{\prime}<\zeta_{1}$

$$
\begin{aligned}
\left|\Delta_{22}\right| & =\left|R_{g}\left(\zeta_{1}, \zeta_{r}, P, \beta\right)-R_{g}\left(\bar{\zeta}_{1}, \zeta_{r}, P, \beta\right)\right| \\
= & \left|\zeta_{1}-\bar{\zeta}_{1}\right| \mid-\zeta_{r} \frac{4 P^{3} \zeta_{1}^{\prime}}{\left(\zeta_{1}^{\prime 2}+P^{2}\right)^{2}}\left(\frac{5}{2}-P^{2}\right) \cos \beta \\
& +\frac{2 P^{3} \zeta_{1}^{\prime}}{\left(\zeta_{1}^{\prime 2}+P^{2}\right)^{2}} \zeta_{r}\left(2 \cos \beta+\frac{\zeta_{r}}{P} \cos ^{2} \beta\right) g\left(\zeta_{1}^{\prime}, \zeta_{r}, P, \beta\right) \mid \\
\leq & \left|\zeta_{1}-\bar{\zeta}_{1}\right| \zeta_{r}\left(4|\cos \beta|\left|\frac{5}{2}-P^{2}\right|+\left(4|\cos \beta|+\zeta_{r} \frac{2 \cos ^{2} \beta}{P}\right)\left|g\left(\zeta_{1}^{\prime}, \zeta_{r}, P, \beta\right)\right|\right) .
\end{aligned}
$$

Similar to the case of $\Delta_{21}$, we use the original form of $\Delta_{22}$ in $\left(0,\left|\zeta_{1}-\bar{\zeta}_{1}\right|^{\frac{1}{2}}\right) \times I$ and the above estimate (A.9) in $\left(\left|\zeta_{1}-\bar{\zeta}_{1}\right|^{\frac{1}{2}}, \infty\right) \times I$ to obtain

$$
\begin{aligned}
& \left|\int_{0}^{2 \pi} \int_{0}^{\infty} \frac{c_{1} P}{\sqrt{\bar{\zeta}_{1}^{2}+P^{2}}} e^{-\frac{\bar{\zeta}_{1}^{2}}{2}-\frac{P^{4}}{\bar{\zeta}_{1}^{2}+P^{2}}} \Delta_{22}\left(1+\frac{P}{\zeta_{r}} \cos \beta\right) d P d \beta\right| \\
\leq & \left|\zeta_{1}-\bar{\zeta}_{1}\right|^{\frac{1}{2}}\left(\left\|g_{7}\right\|_{L^{1}}+\left\|g_{4}\left(\zeta_{1}, \zeta_{r}, \cdot, \cdot\right)\right\|_{L^{\infty}\left(0, \frac{1}{10}\right) \times I}+\left\|g_{4}\left(\bar{\zeta}_{1}, \zeta_{r}, \cdot, \cdot\right)\right\|_{L^{\infty}\left(0, \frac{1}{10}\right) \times I}\right) \\
& +\left|\zeta_{1}-\bar{\zeta}_{1}\right|\left(\left\|g_{5}\right\|_{L^{1}}+\left\|g_{6}\right\|_{L^{1}}\right),
\end{aligned}
$$

where

$$
\begin{aligned}
& g_{5}=4 c_{1}\left|\cos \beta \| \frac{5}{2}-P^{2}\right| e^{-\frac{P^{4}}{\bar{\zeta}_{1}^{2}+P^{2}}}\left(\zeta_{r}+P|\cos \beta|\right), \\
& g_{6}=4 c_{1} e^{-\frac{P^{4}}{\zeta_{1}^{2}+P^{2}}}|\cos \beta|\left(\zeta_{r}+P|\cos \beta|\right) g\left(\zeta_{1}^{\prime}, \zeta_{r}, P, \beta\right), \\
& g_{7}=2 \zeta_{r} c_{1} e^{-\frac{P^{4}}{\zeta_{1}^{2}+P^{2}}}|\cos \beta|^{2}\left(\zeta_{r}+P|\cos \beta|\right) g\left(\zeta_{1}^{\prime}, \zeta_{r}, P, \beta\right) .
\end{aligned}
$$

We have already known that $\left\|g_{4}\right\|_{L^{\infty}\left(0, \frac{1}{10}\right) \times I}$ is bounded. For $g_{5}$, from (A.10) we have

$$
\left|g_{5}\right| \leq \begin{cases}4 c_{1}|\cos \beta|\left(\frac{5}{2}-P^{2}\right)(1+P|\cos \beta|), & \text { for } 0<P<1, \\ 4 c_{1}|\cos \beta|\left(\frac{5}{2}+P^{2}\right)(1+P|\cos \beta|) e^{-\frac{10000}{10001} P^{2}}, & \text { for } 1<P .\end{cases}
$$


For $g_{6}$, using $\zeta_{1}^{\prime} \geq \bar{\zeta}_{1}$ and (4.7), we have from the definition (A.11)

$$
\begin{aligned}
\left|g_{6}\right| \leq 4 c_{1}|\cos \beta| & \left(\zeta_{r}+P|\cos \beta|\right) \\
\cdot & \left|\frac{5}{2}-P^{2}-\zeta_{r}^{2}-2 P \zeta_{r} \cos \beta\right| e^{-\frac{P^{2}}{\zeta_{1}^{\prime 2}+P^{2}}\left(P+\zeta_{r} \cos \beta\right)^{2}} .
\end{aligned}
$$

From this we have

$$
\left|g_{6}\right| \leq\left\{\begin{array}{cl}
10 c_{1}|\cos \beta|(1+P|\cos \beta|), & \text { for } 0<P<1, \\
4 c_{1}|\cos \beta|(1+P|\cos \beta|) & \\
\cdot\left(\frac{7}{2}+P^{2}+2 P|\cos \beta|\right) e^{-\frac{10000}{10001}(P-1)^{2}}, & \text { for } P>1 .
\end{array}\right.
$$

In the same way, for $g_{7}$ we have from its definition (A.12)

$$
\begin{aligned}
&\left|g_{7}\right| \leq 2 c_{1} \cos ^{2} \beta\left(\zeta_{r}+P|\cos \beta|\right) \\
& \cdot\left|\frac{5}{2}-P^{2}-\zeta_{r}^{2}-2 P \zeta_{r} \cos \beta\right| e^{-\frac{P^{2}}{\zeta_{1}^{2}+P^{2}}\left(P+\zeta_{r} \cos \beta\right)^{2}},
\end{aligned}
$$

and thus

$$
\left|g_{7}\right| \leq\left\{\begin{array}{cl}
5 c_{1} \cos ^{2} \beta(1+P|\cos \beta|), & \text { for } 0<P<1, \\
2 c_{1} \cos ^{2} \beta(1+P|\cos \beta|) & \\
\cdot\left(\frac{7}{2}+P^{2}+2 P|\cos \beta|\right) e^{-\frac{10000}{10001}(P-1)^{2}}, & \text { for } 1 \leq P .
\end{array}\right.
$$

From these estimates, we see that $\left\|g_{7}\right\|_{L^{1}},\left\|g_{5}\right\|_{L^{1}}$, and $\left\|g_{6}\right\|_{L^{1}}$ are bounded. This finishes the proof of (4.17) for $0 \leq \zeta_{r} \leq 1$.

Next, we turn to the proof of (4.18) for $0 \leq \zeta_{r} \leq 1$. Consider $H_{1}$ part first. By the mean value theorem, for some $\bar{\zeta}_{1}<\tilde{\zeta}_{1}<\zeta_{1}$ we have

$$
\begin{aligned}
& \left|H_{1}\left(\zeta_{1}\right)-H_{1}\left(\bar{\zeta}_{1}\right)\right| \\
\leq & \left|\zeta_{1}-\bar{\zeta}_{1}\right| \int_{0}^{\infty}\left|\frac{2 \pi c_{2} P}{\sqrt{\tilde{\zeta}_{1}^{2}+P^{2}}} \tilde{\zeta}_{1}\left(1-\tilde{\zeta}_{1}^{2}-P^{2}\right)\left(\frac{5}{2}-\frac{9}{2} P^{2}+P^{4}\right) e^{-\frac{\tilde{\zeta}_{1}^{2}}{2}-P^{2}}\right| d P \\
\leq & \left|\zeta_{1}-\bar{\zeta}_{1}\right| \int_{0}^{\infty} \frac{1}{50} \pi c_{2}\left(1+P^{2}\right)\left(\frac{5}{2}+\frac{9}{2} P^{2}+P^{4}\right) e^{-P^{2}} d P \leq C\left|\zeta_{1}-\bar{\zeta}_{1}\right| .
\end{aligned}
$$


Here we have used the fact that $0 \leq \tilde{\zeta}_{1} \leq \frac{1}{100}$.

For $H_{2}$ part, by the mean value theorem, for some $\bar{\zeta}_{1}<\zeta_{1}^{\prime}<\zeta_{1}$ we have

$$
\begin{aligned}
& \left|H_{2}\left(\zeta_{1}, \zeta_{r}\right)-H_{2}\left(\bar{\zeta}_{1}, \zeta_{r}\right)\right| \\
\leq & \left|\zeta_{1}-\bar{\zeta}_{1}\right| \int_{0}^{\infty} \int_{0}^{2 \pi} c_{2} P\left|\left(1+\frac{P}{\zeta_{r}} \cos \beta\right) R_{h} \frac{1-\zeta_{1}^{2}-P^{2}}{\sqrt{\zeta_{1}^{2}+P^{2}}} \zeta_{1} e^{-\frac{\zeta_{1}^{2}}{2}-P^{2}}\right|_{\zeta_{1}=\zeta_{1}^{\prime}} d \beta d P \\
= & \left|\zeta_{1}-\bar{\zeta}_{1}\right| \int_{0}^{\infty} \int_{0}^{2 \pi}\left|h_{1}+h_{2}\right| d P d \beta,
\end{aligned}
$$

where

$$
\begin{aligned}
& h_{1}=c_{2} \zeta_{1}^{\prime} \frac{P}{\sqrt{\zeta_{1}^{\prime 2}+P^{2}}}\left(1-\zeta_{1}^{\prime 2}-P^{2}\right) e^{-\frac{\zeta_{1}^{2}}{2}-P^{2}} R_{h}\left(\zeta_{r}, P, \beta\right), \\
& h_{2}=c_{2} \zeta_{1}^{\prime} \frac{P}{\sqrt{\zeta_{1}^{\prime 2}+P^{2}}}\left(1-\zeta_{1}^{\prime 2}-P^{2}\right) P \cos \beta e^{-\frac{\zeta_{1}^{\prime 2}}{2}-P^{2}} \frac{1}{\zeta_{r}} R_{h}\left(\zeta_{r}, P, \beta\right) .
\end{aligned}
$$

Note that, from (4.12) and (4.8), we have

$$
\begin{aligned}
\frac{1}{\zeta_{r}} R_{h}\left(\zeta_{r}, P, \beta\right)= & -\frac{1}{\zeta_{r}}\left(\frac{5}{2}-P^{2}\right)\left(1-e^{-\frac{3}{2} \zeta_{r}^{2}-2 \zeta_{r} P \cos \beta}\right) \\
& -\left(\zeta_{r}+2 P \cos \beta\right) e^{-\frac{3}{2} \zeta_{r}^{2}-2 \zeta_{r} P \cos \beta}+2 P \cos \beta\left(\frac{7}{2}-P^{2}\right) \\
= & -\left(\frac{5}{2}-P^{2}\right)\left(3 \zeta_{r}^{\prime}+2 P \cos \beta\right) e^{-\frac{3}{2} \zeta_{r}^{\prime 2}-2 \zeta_{r}^{\prime} P \cos \beta} \\
& -\left(\zeta_{r}+2 P \cos \beta\right) e^{-\frac{3}{2} \zeta_{r}^{2}-2 \zeta_{r} P \cos \beta}+2 P \cos \beta\left(\frac{7}{2}-P^{2}\right),
\end{aligned}
$$

for some $0<\zeta_{r}^{\prime}<\zeta_{r}$ by the mean value theorem and that

$$
-\frac{3}{2} \zeta_{r}^{2}-2 P \zeta_{r} \cos \beta-P^{2}=-\frac{3}{2} \zeta_{r}^{2} \sin ^{2} \beta-\frac{3}{2}\left(\zeta_{r} \cos \beta+\frac{2}{3} P\right)^{2}-\frac{1}{3} P^{2}
$$

Taking these into account, we have

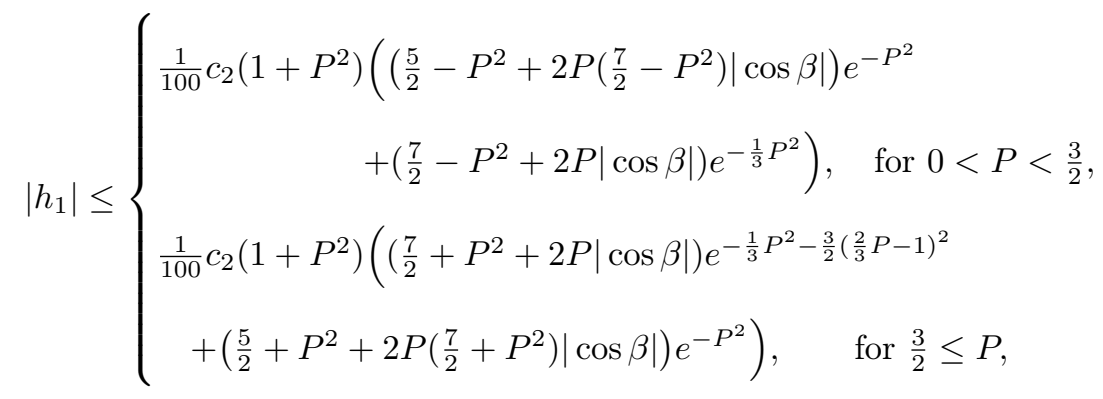




$$
\left|h_{2}\right| \leq\left\{\begin{array}{cc}
\frac{1}{100} c_{2}\left(1+P^{2}\right) P|\cos \beta|\left[\left(\left(\frac{5}{2}-P^{2}\right)(3+2 P|\cos \beta|)\right.\right. \\
\left.+1+2 P|\cos \beta|) e^{-\frac{1}{3} P^{2}}+2 P\left(\frac{7}{2}-P^{2}\right)|\cos \beta| e^{-P^{2}}\right], \\
\frac{1}{100} c_{2}\left(1+P^{2}\right) P|\cos \beta|\left[\left(\left(\frac{5}{2}+P^{2}\right)(3+2 P|\cos \beta|)\right.\right. \\
+1+2 P|\cos \beta|) e^{-\frac{1}{3} P^{2}-\frac{3}{2}\left(\frac{2}{3} P-1\right)^{2}} & \text { for } 0<P<\frac{3}{2}, \\
\left.+2 P\left(\frac{7}{2}+P^{2}\right)|\cos \beta| e^{-P^{2}}\right], & \text { for } \frac{3}{2} \leq P .
\end{array}\right.
$$

From these estimates, we see that $\left\|h_{1}+h_{2}\right\|_{L^{1}}$ is bounded and conclude

$$
\left|H_{2}\left(\zeta_{1}, \zeta_{r}\right)-H_{2}\left(\bar{\zeta}_{1}, \zeta_{r}\right)\right| \leq C\left|\zeta_{1}-\bar{\zeta}_{1}\right|
$$

Combining (A.13) and (A.14) yields (4.18) for $0 \leq \zeta_{r} \leq 1$ in Lemma 4.2.

Acknowledgments. The research of I-Kun Chen is supported in part by the Institute of Mathematics, Academia Sinica, Taipei, Department of Applied Mathematics, National Chiao Tung University, Hsinchu, and NSC Grant 1012115M009015MY2; that of Hitoshi Funagane and Shigeru Takata by KAKENHI from JSPS (No. 23656068); and that of Tai-Ping Liu by National Science Foundation Grant DMS 0709248 and National Science Council Grant 96-2628-M-001-011.

\section{References}

1. Abramowitz, M.; Stegun, I. A.: Handbook of Mathematical Functions: With Formulas, Graphs, and Mathematical Tables, Courier Dover Publications, 1964.

2. Chen, C.-C.; Chen, I-K.; Liu, T.-P.; Sone, Y.: Thermal transpiration for the linearized Boltzmann equation. Comm. Pure Appl. Math. 60 (2007), 147-163 .

3. Chen, I-K.; Liu, T.-P.; Takata, S.: Boundary singularity for thermal transpiration problem of the linearized Boltzmann equation. Arch. Ration. Mech. Anal. 212 (2014), no. 2, 575595. 
4. Ohwada, T.; Sone, Y.; Aoki, K.: Numerical analysis of the Poiseuille and thermal transpiration flows between two parallel plates on the basis of the Boltzmann equation for hard-sphere molecules. Phys. Fluids A 1 (1989), 2042-2049.

5. Sone, Y.: Molecular Gas Dynamics. Theory, Techniques, and Applications, Birkhäuser, Boston, 2007.

6. Takata, S.; Funagane, H.: Poiseuille and thermal transpiration flows of a highly rarefied gas: over-concentration in the velocity distribution function. J. Fluid Mech. 669 (2011), $242-259$.

7. Takata, S.; Funagane, H.: Singular behaviour of a rarefied gas on a planar boundary. J. Fluid Mech. 717 (2013), 30-47. 